\title{
The Ameliorative Effects of Arctiin and Arctigenin on the Oxidative Injury of Lung Induced by Silica via TLR-4/NLRP3/TGF- $\beta$ Signaling Pathway
}

\author{
Xueying Liu $\mathbb{D}^{1},{ }^{1}$ Jian Wang $\left(\mathbb{D},{ }^{2}\right.$ Peiyuan Dou $\left(\mathbb{D},{ }^{1}\right.$ Xu Zhang $\mathbb{D}^{1},{ }^{1}$ Xiaoku Ran $\left(\mathbb{D},{ }^{1}\right.$ Linlin Liu $(\mathbb{D})$, \\ and Deqiang Dou ${ }^{1}{ }^{1}$ \\ ${ }^{1}$ College of Pharmacy, Liaoning University of Traditional Chinese Medicine, Dalian 116600, China \\ ${ }^{2}$ Department of Medicinal Chemistry, Shenyang Pharmaceutical University, Shenyang 110032, China \\ Correspondence should be addressed to Deqiang Dou; deqiangdou@126.com
}

Received 5 February 2021; Revised 18 May 2021; Accepted 26 June 2021; Published 19 July 2021

Academic Editor: Jayeeta Ghose

Copyright (C) 2021 Xueying Liu et al. This is an open access article distributed under the Creative Commons Attribution License, which permits unrestricted use, distribution, and reproduction in any medium, provided the original work is properly cited.

\begin{abstract}
Silicosis remains one of the most serious diseases worldwide, with no effective drug for its treatment. Our research results have indicated that arctiin and arctigenin could increase the mitochondrial membrane potential, which in turn reduces the production of reactive oxygen species (ROS), blocks the polarization of macrophages, and inhibits the differentiation of myofibroblasts to reduce oxidative stress, inflammation, and fibrosis. Further, our study revealed that arctiin and arctigenin suppressed the activation of NLRP3 inflammasome through the TLR-4/Myd88/NF- $\kappa$ B pathway and the silica-induced secretion of TNF- $\alpha$, IL- $1 \beta$, TGF- $\beta$, and $\alpha$-SMA. Besides, the silica-induced increase in the levels of serum ceruloplasmin and HYP was also inhibited. Results of metabolomics indicated that arctiin and arctigenin could regulate the abnormal metabolic pathways associated with the development of silicosis, which involve pantothenate and CoA biosynthesis, cysteine and methionine metabolism, linoleic acid metabolism, and arginine and proline metabolism successively. Furthermore, the analysis of metabolomics, together with network topological analysis in different phases of silicosis, revealed that urine myristic acid, serum 4-hydroxyproline, and L-arginine could be regarded as diagnosis biomarkers in the early phase and formation of pulmonary fibrosis in the latter phases of silicosis. Arctiin and arctigenin could downregulate the increased levels of myristic acid in the early phase and serum 4-hydroxyproline in the latter phase of silicosis. Interestingly, the integration of TLR-4/NLRP3/TGF- $\beta$ signaling and metabolomics verified the importance of macrophage polarization in the silicosis fibrosis process. To the best of our knowledge, this is the first study reporting that arctiin and arctigenin both can ameliorate silicosis effectively, and the former is a little stronger than its aglycone arctigenin because of its high oral bioavailability, low toxicity, and multimolecular active metabolites as determined by AdmetSAR and molecular docking analysis.
\end{abstract}

\section{Introduction}

Silicosis is an occupational disease characterized by chronic lung inflammation, progressive pulmonary fibrosis, and systemic immune dysfunction [1-3]. The innate and adaptive immune systems are regarded to play key regulatory roles in early phases of silicosis [4]. Previous studies have indicated that silica can damage macrophages, activate the innate immune system, and initiate an inflammatory response in the lungs. As silicosis progresses, fibrosis and inflammation occur together; oxygen- and nitrogen-derived free radicals play a major role in silica-induced lung injury and fibrosis [5]. Silicosis is usually ignored or misdiagnosed as other diseases because only inflammation occurs at its early phase. Presently, X-ray is the primary method for diagnosing silicosis, but it is not suitable for early diagnosis as the image shadows only show the fibrosis formation. Fibrosis can lead to overdeposition of extracellular matrix, and ultimately lead to structural reconstitution of lung tissue and even respiratory failure. The mortality rate after lung fibrosis generally 
accounts for $50 \%$ to $70 \%[6,7]$. It is well known that it is difficult to recover after fibrosis; thus, early diagnosis and treatment for silicosis are important.

Metabolomics can comprehensively reflect changes in the metabolites of organisms in different states. The measurement of metabolite levels and variations in biofluids can offer many insights into disease processes and response to therapeutic intervention [8]. Urine is one of the terminal products of organisms, and its collection is simple and noninvasive to the body. Urine metabolites can reflect metabolism disorders and provide insights into the responses of the body to physiological dynamic changes or disease processes [8-10]. In addition, the composition and content of urine metabolites can also reflect changes in the body's metabolic network under the influence of diseases and drugs, and these attributes are essential for addressing the challenges associated with biomarker discovery [8]. Network pharmacology, as a newly developed strategy, focuses on searching for the relationships between compounds and their potential targets, and can help elucidate the molecular mechanism of action in metabolomics [11]. Moreover, molecular docking could be used not only to screen the action target and mechanism of drugs but also to provide the probable binding ability of drugs to the target [12].

Fructus Arctii, the dried fruit of Arctium lappa L., has the function of clearing away heat and nourishing the lung as recorded in China's Pharmacopoeia [13]. Arctiin is its main active component and accounting for over 5\% of Fructus Arctii as stipulated in Chinese Pharmacopoeia [13]. Research indicated that arctigenin has the function of antioxidation, anti-inflammation [14, 15], antitumor [16], and neuronprotective activities $[17,18]$. Recent studies have shown that arctigenin can also suppress renal interstitial fibrosis, repress TGF- $\beta$-induced epithelial-mesenchymal transition (EMT) in human lung cancer cells, and attenuate PQ-induced EMT and pulmonary fibrosis [19-21]. Arctiin has also been reported to possess some similar biological functions [2226]. However, the content of arctigenin in Fructus Arctii is much lower than that of arctiin. In our previous research, we found that arctiin could be metabolized into arctigenin in the intestinal tract $[27,28]$, and arctigenin was regarded to be the active form of arctiin. Based on these findings, we hypothesized that arctiin and arctigenin could ameliorate silica-induced oxidative injury in the lungs.

Herein, urine metabolomics was applied to explore the progress of silicosis with the aim at finding stable bioindices for the early diagnosis of silicosis and investigating the metabolic pathways in different phases of silicosis. Besides, the effects of arctiin and arctigenin on the progress of silicosis were compared to determine the more suitable compound for treatment. Network pharmacology and molecular docking were used to predict and analyze potential mechanisms for providing a theoretical basis for the development and utilization of arctiin and its metabolites.

\section{Materials and Methods}

2.1. Animals and Treatment. Adult male SPF level Wistar rats (weighing approximately 180-220 g) were obtained from the
Liaoning Changsheng Biotechnology Co. Ltd., Liaoning, China (license key: SCXK (Liao) 20150001), housed in a specific pathogen-free environment, and maintained under controlled conditions $\left(22 \pm 2^{\circ} \mathrm{C}, 40 \pm 10 \%\right.$ relative humidity, and $12 \mathrm{~h}$ light/dark cycle) with free access to standard food and water. Animal research was approved by the Animal Ethical and Welfare Committee of Liaoning University of Traditional Chinese Medicine, and the experimental procedures were conducted according to the Guide for Care and Use of Laboratory Animals of Liaoning University of Traditional Chinese Medicine. The rats were modeled by tracheal intubation with $0.5 \mathrm{~mL}$ of $80 \mathrm{mg} / \mathrm{mL} \mathrm{SiO}_{2}$ suspension $(4000 \mathrm{U} / \mathrm{mL}$ penicillin sodium), and the model rats were evaluated via serum hydroxyproline and histopathological analysis. As described in Figure 1(a), after modeling, the rats in different groups were continuously intragastrically administrated with the corresponding drugs for 30 days. The rats were randomly divided into nine groups: the control group (CON, solvent), the model group (MOD, solvent), the tetrandrine-positive control group (POS, $30 \mathrm{mg} / \mathrm{kg}$ ), the low-dose arctiin group (ACL, $30 \mathrm{mg} / \mathrm{kg}$ ), the high-dose arctiin group (ACH, $60 \mathrm{mg} / \mathrm{kg}$ ), the low-dose arctigenin group (AGL, $30 \mathrm{mg} / \mathrm{kg}$ ), the high-dose arctigenin group (AGH, $60 \mathrm{mg} / \mathrm{kg}$ ), the arctiin + positive group (ACP, arctiin $30 \mathrm{mg} / \mathrm{kg}$ and tetrandrine $30 \mathrm{mg} / \mathrm{kg}$ ), and the arctigenin + positive group (AGP, arctigenin $30 \mathrm{mg} / \mathrm{kg}$ and tetrandrine $30 \mathrm{mg} / \mathrm{kg}$ ). Further details of modeling and treatment are shown in Supplemental Materials.

2.2. Animal Behavior, Histological Assessment, and Mediator Measurements. Mental state, body weight, fur luster, respiratory rate, urine volume, dietary amount, and the amount of drinking water were monitored every week according to the methods specified in Supplemental Materials. Lung, spleen, and thymus tissues were collected and weighed to calculate their coefficient after drawing blood samples. Following this, the right part of the lung was used for the determination of bioindices and the left part was fixed in $4 \%$ paraformaldehyde solution, embedded in paraffin, and sectioned at a thickness of $5 \mu \mathrm{m}$ for histological evaluation via hematoxylin and eosin (H\&E) and Masson's staining. The levels of hydroxyproline, ceruloplasmin, and lysozyme in the serum and several proinflammatory and profibrosis cytokines in lung tissues, including TNF- $\alpha$, IL- $1 \beta, \mathrm{NF}-\kappa \mathrm{B}$, and TGF- $\beta$, were determined by ELISA, and the expression levels of TLR-4, Myd88, NF- $\kappa$ B p65, NLRP3, ASC, cleaved caspase1 , and $\alpha$-SMA were assayed by Western blotting, aiming at evaluating the mechanism of arctiin and arctigenin against silicosis. Further details have been provided in Supplemental Materials.

2.3. Metabolomics Analysis. To explore the endogenous changes in the progression of silicosis, metabolomics was analyzed by HPLC-QTOF-MS (Agilent, USA), and data were preprocessed using MetaboAnalyst 4.0 (https://www .metaboanalyst.ca/).The normalized data were then subjected to Orthogonal Projections to Latent Structures Discriminant Analysis (OPLS-DA) in a Simca-p 14.1 workstation. Metabolites with VIP $>1$ and $P<0.05$ were identified as differential 


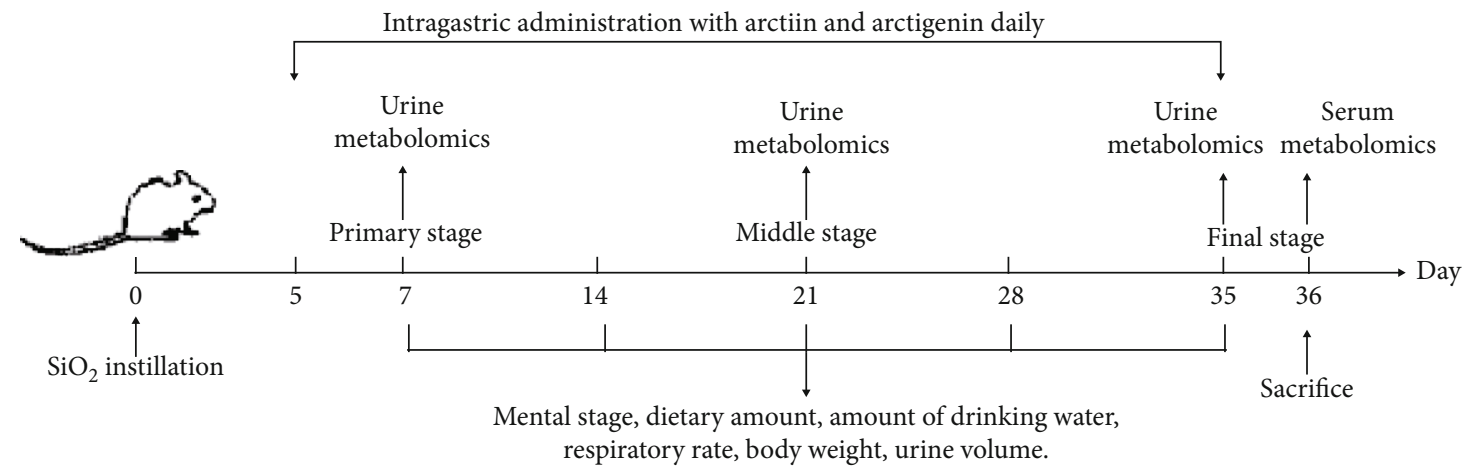

(a)

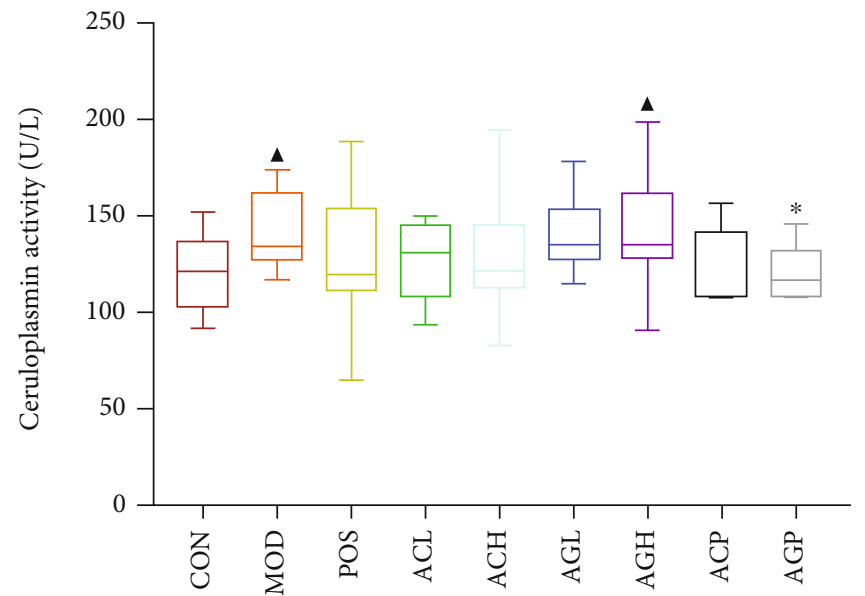

(b)

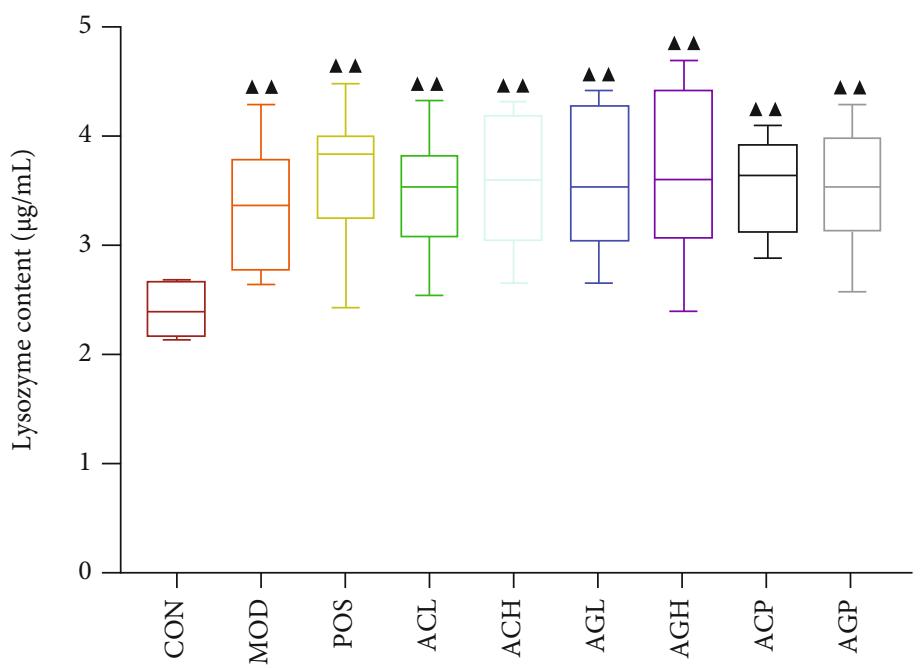

(c)

Figure 1: Continued. 


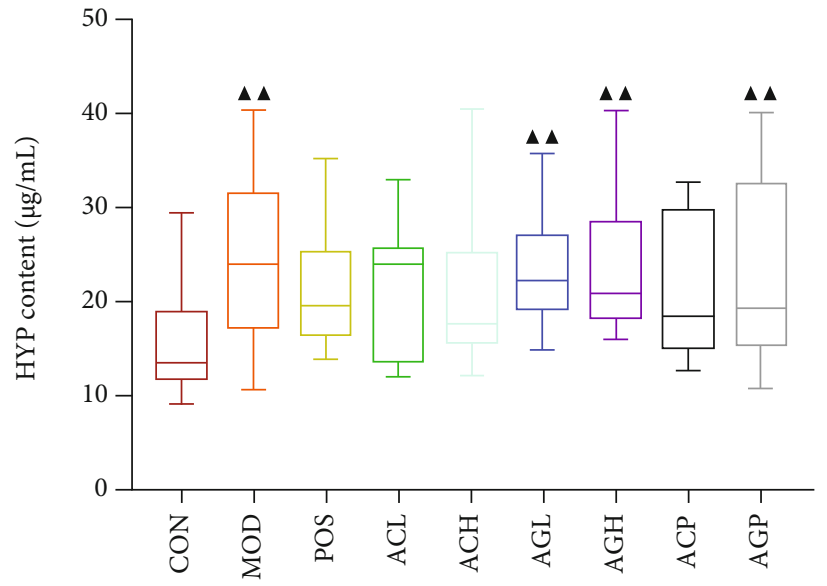

(d)

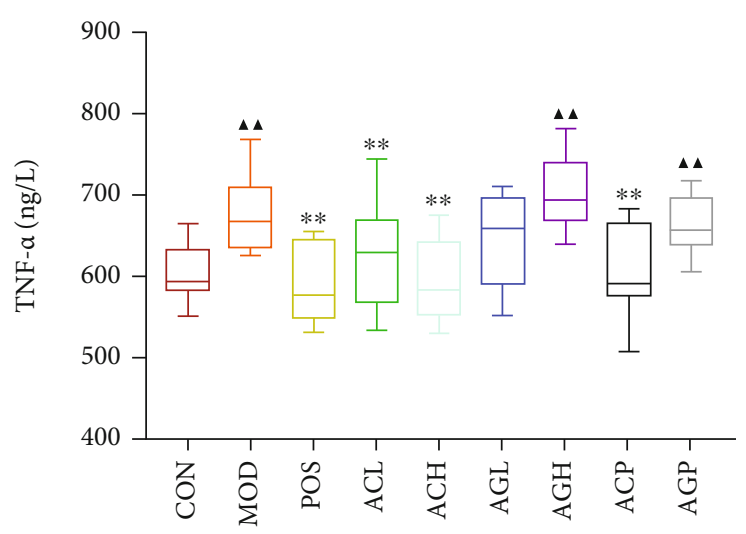

(e)

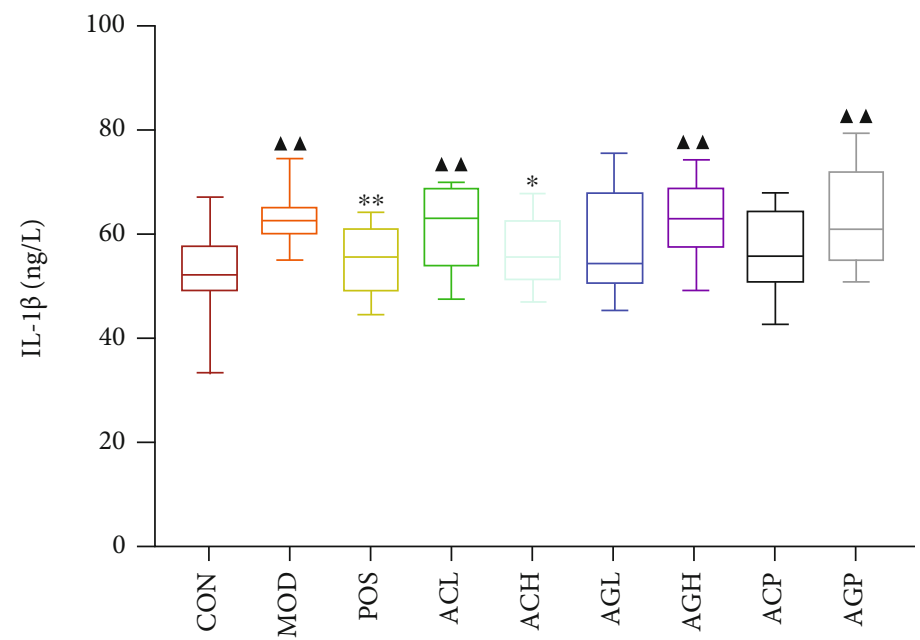

(f)

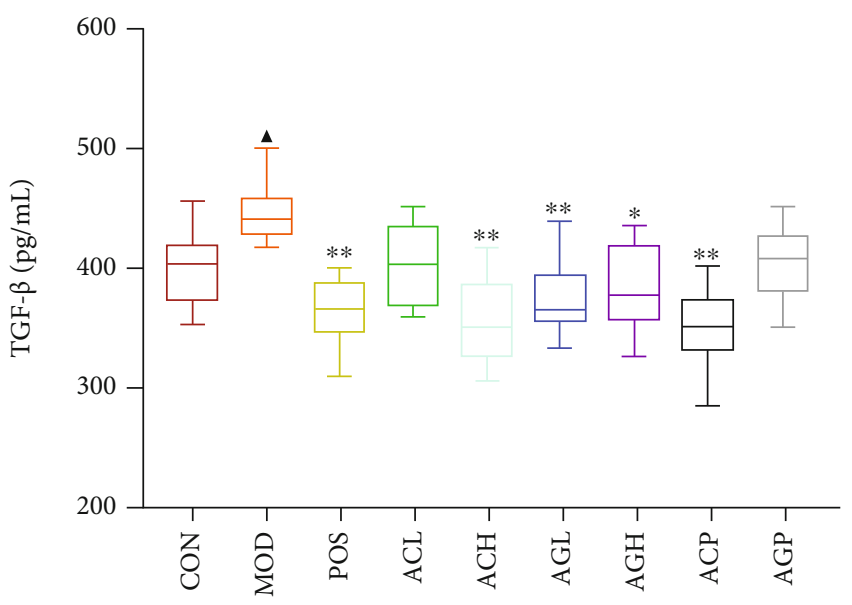

(g)

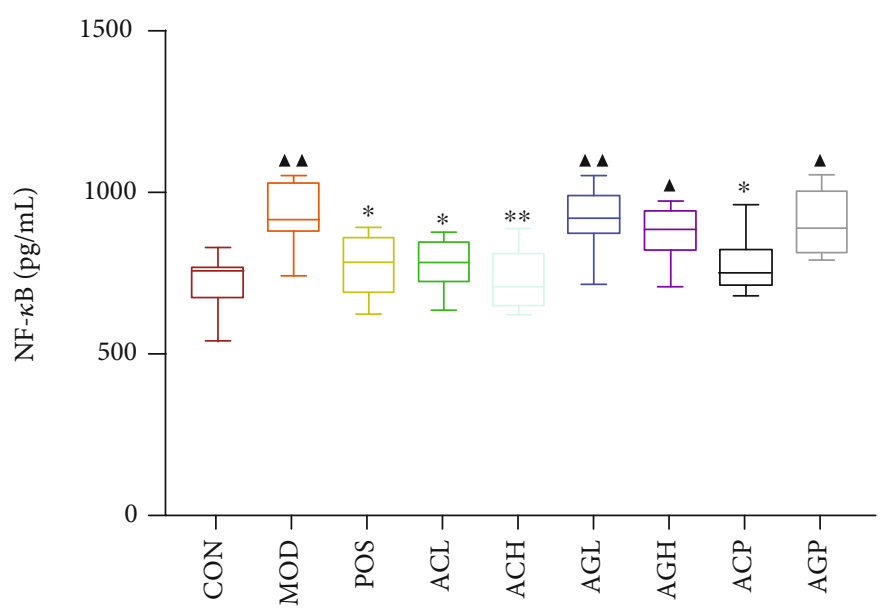

(h)

FIgURe 1: Continued. 


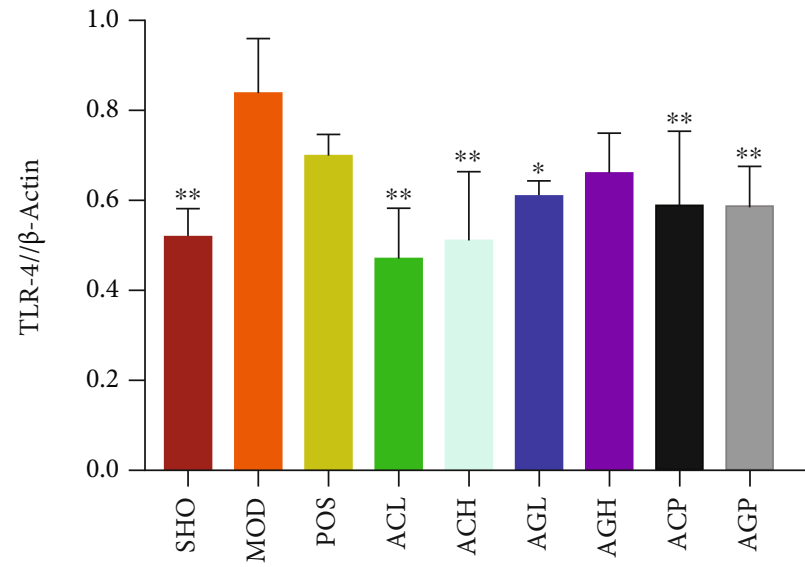

(i)

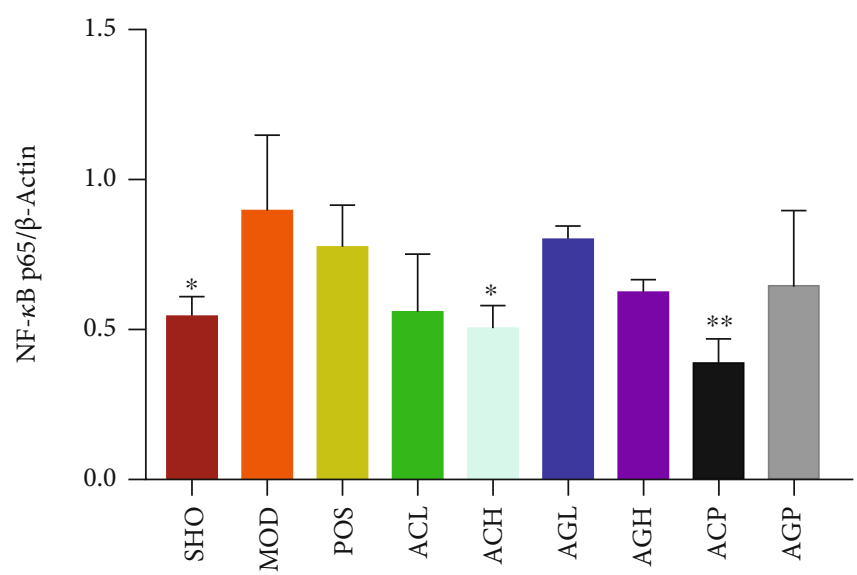

(k)

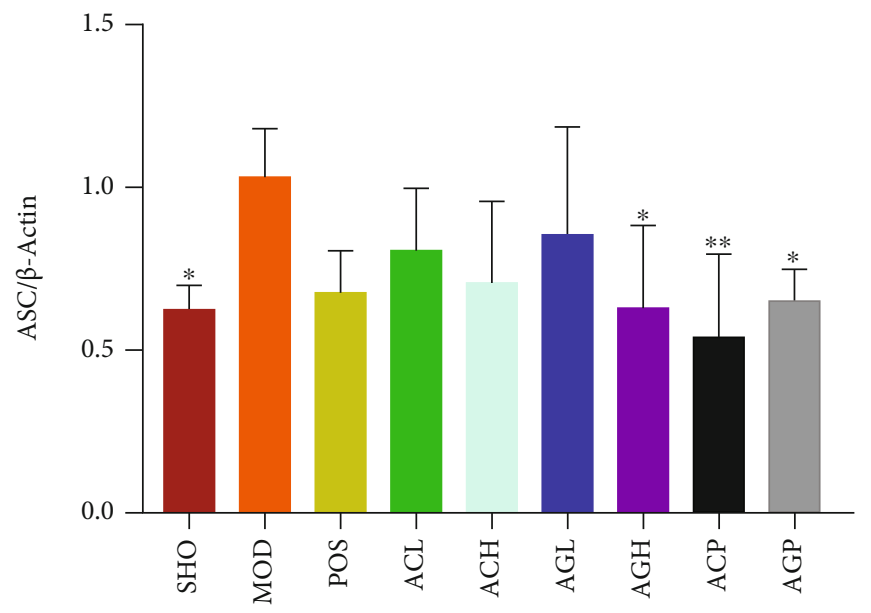

(m)

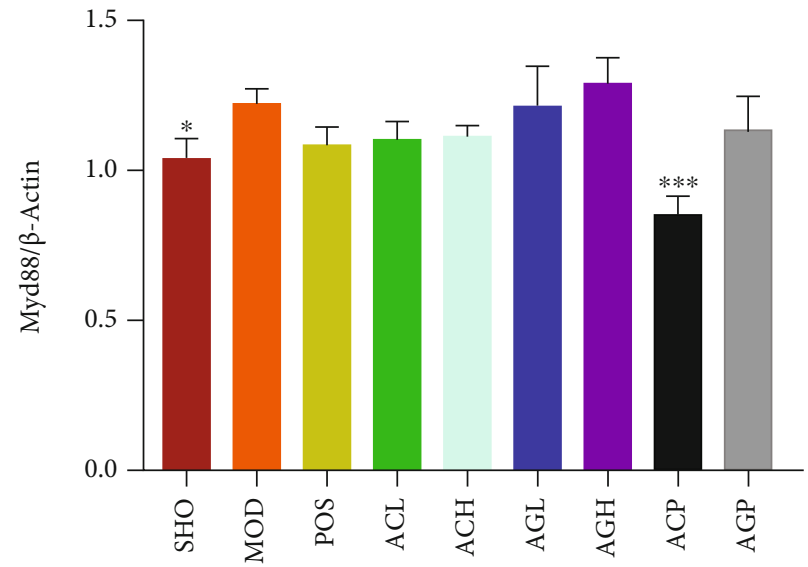

(j)

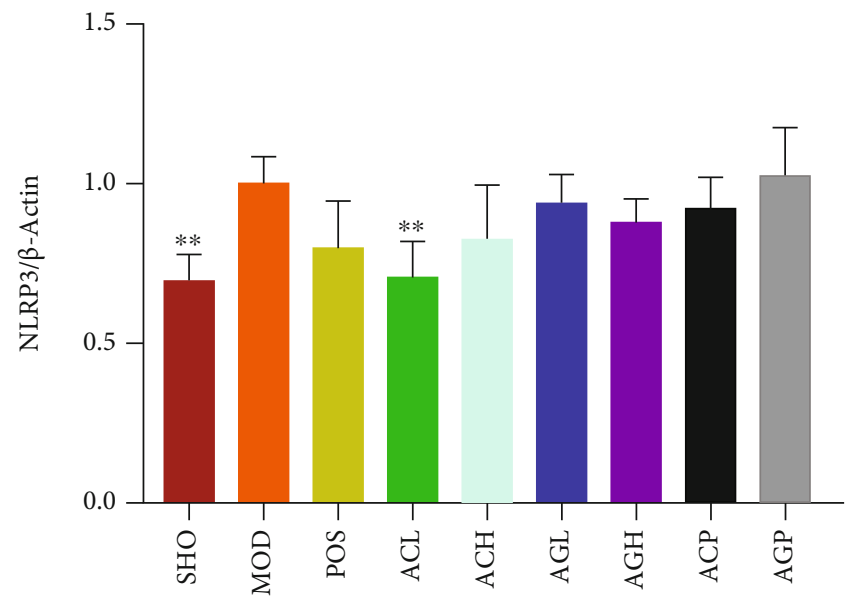

(1)

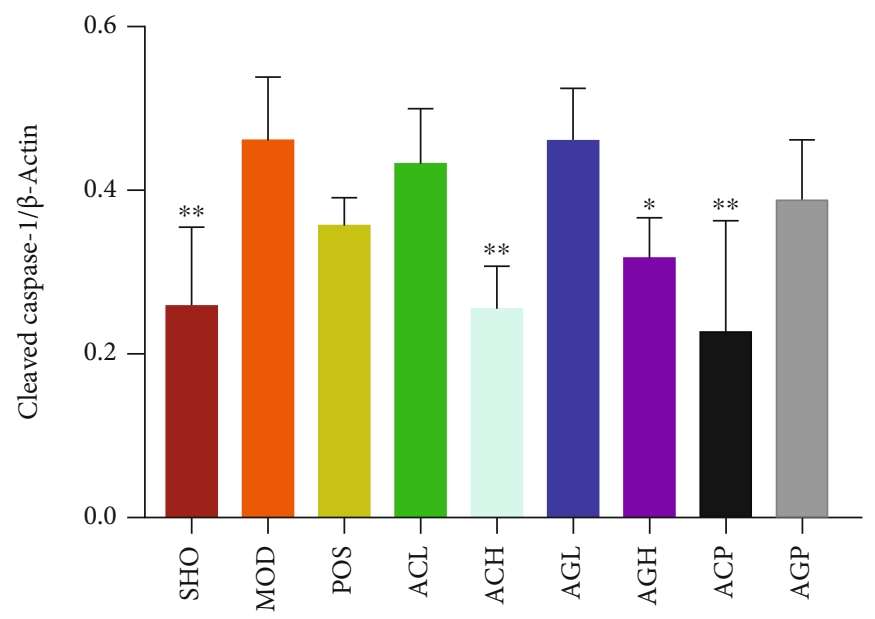

(n)

Figure 1: Continued. 


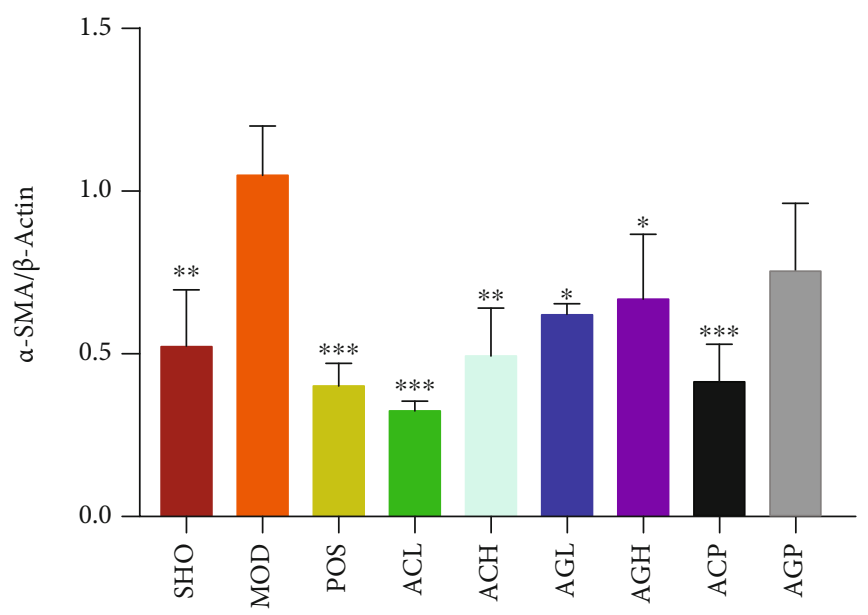

(o)

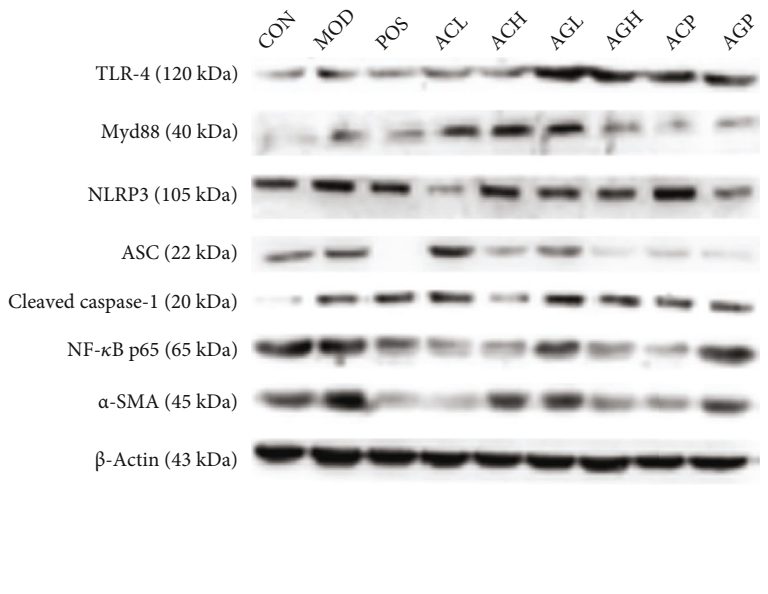

(p)

FIGURE 1: Experimental progress (a). The activities of ceruloplasmin (b) and lysozyme (c) in serum. The content of serum hydroxyproline (d). The level of TNF- $\alpha$, IL- $1 \beta$, TGF- $\beta$, and NF- $\kappa$ B in lung tissues measured by ELISA (e-h). Note: mean \pm SD, $n=8 .{ }^{\mathbf{\Delta}} P<0.05$ and ${ }^{\mathbf{\Delta}} P<0.01$ compared with CON. ${ }^{*} P<0.05$ and ${ }^{* *} P<0.01$ compared with MOD. The expression of TLR- 4 , NLRP3, Myd88, ACS, NF- $\kappa$ B, and $\alpha$-SMA in lung tissues measured by Western blot (i-p). Note: mean $\pm \mathrm{SD}, n=3 .{ }^{*} P<0.05,{ }^{* *} P<0.01$, and ${ }^{* * *} P<0.001$ compared with MOD.

metabolites, and compound validation was conducted to verify the accuracy and eliminate unreliable differential metabolites. The metabolic pathways were identified by analyzing and enriching differential metabolites screened using the Pathway Analysis module in MetaboAnalyst 4.0. The MetScape plugin in Cytoscape 3.7.1 software was used for network construction of different periods and analysis of the topology of each network. Euclidean distance was used to evaluate the similarity between different periods by network topology and to determine the important metabolites in various periods [29]. A Venn diagram of metabolites in each period was displayed using the FunRich software, and changes in the same metabolite in different periods were analyzed. The diagnostic value was predicted using the ROC-based Biomarker Analysis module in MetaboAnalyst 4.0. Further details are presented in Supplemental Materials.

\subsection{Effects of Arctiin and Arctigenin on Silica-Induced} Macrophage Inflammation. To verify the effects of arctiin and arctigenin against inflammation, RAW264.7 murine macrophage cells were obtained from the Cell Bank of the Chinese Academy of Sciences (Shanghai, China). The cytotoxicity of arctiin and arctigenin toward RAW264.7 cells exposed to silica $(50 \mu \mathrm{g} / \mathrm{mL})$ was assayed by MTT. The cells were then seeded in a 6 -well plate at $1 \times 10^{6}$ cells per well and incubated in DMEM containing $10 \%$ FBS for $24 \mathrm{~h}$. The cells of the arctiin group (ARC) and the arctigenin group (ARG) were treated with $1 \mu \mathrm{M}$ arctiin or arctigenin and silica $(50 \mu \mathrm{g} / \mathrm{mL})$ together for $24 \mathrm{~h}$. The mitochondrial membrane potential and reactive oxygen species were detected using the assay kit, and the expression levels of iNOS, Arg-1, TLR-4, NLRP3, cleaved caspase- 1 , and TGF- $\beta$ were evaluated. Further details are shown in Supplemental Materials.

2.5. Effects of Arctiin and Arctigenin on TGF- $\beta 1$-Induced Myofibroblast Differentiation. To verify the effects of arctiin and arctigenin against fibrosis, primary mouse lung fibro- blasts (PLFs) were isolated as described in Supplemental Materials. The cytotoxicity of arctiin and arctigenin on PLFs was assayed by MTT. The cells were seeded in a 6-well plate at $1.5 \times 10^{5}$ cells per well and incubated in DMEM containing $10 \% \mathrm{FBS}$ for $24 \mathrm{~h}$. The cells were then treated with TGF- $\beta 1$ ( $10 \mathrm{ng} / \mathrm{mL})$ for $48 \mathrm{~h}$. The cells were incubated with arctiin and arctigenin at $1 \mu \mathrm{M}$. Following this, the expression level of $\alpha$-SMA was measured by Western blotting. Additional details have been provided in Supplemental Materials.

2.6. Network Pharmacology-Predicted Pathway. Potential targets of arctiin and arctigenin were predicted by using TCMSP (http://lsp.nwu.edu.cn/tcmsp.php), STITCH (http://stitch .embl.de/), and SwissTargetPrediction server (http://www .swisstargetprediction.ch/), with stunted Homo sapiens as organism. CTD (https://ctdbase.org/) and GeneCards (https://www.genecards.org/) databases were used to find relevant target genes of pulmonary fibrosis. The compounddisease-target gene networks were constructed using the merge function of Cytoscape 3.7.1 software. Subsequently, the PPI network was established by STRING (https://string$\mathrm{db} .0 \mathrm{rg} /$ ) to select the closely connected genes for biological function analysis through FunRich software. The DAVID database (https://david.ncifcrf.gov/tools.jsp) was used to enrich the KEGG and GO pathway analyses of closely connected genes, and the enriched pathways were visualized on the OmicShare cloud platform (http://www.omicshare.com/ forum/).

2.7. Molecular Docking Studies. For the preparation of the protein and the ligand, the crystal structure of TGF- $\beta$ RI complexed with a 2-aminoimidazole inhibitor (PDB ID: 3 faa) was downloaded from the Protein Data Bank (PDB), USA (https://www.rcsb.org/). Protein preparation was conducted using Discovery Studio 3.0 (BIOVIA, San Diego, California, USA); the ions, water molecules, and all internal ligands were removed; the missing atoms were inserted; and 
cocrystallization of small molecules was optimized before minimization of the target protein was conducted. The SDF documents of ligand compounds were downloaded from PubChem (https://pubchem.ncbi.nlm.nih.gov/), the initial molecular structure was optimized using Discovery Studio 3.0, and the molecular docking study was conducted using CDOCKER from Discovery Studio 3.0.

2.8. Prediction of Oral Bioavailability. AdmetSAR2.0 (http:// Immd.ecust.edu.cn/admetsar2/) was used to predict the oral bioavailability according to the Morgan fingerprint and random forest methods.

2.9. Statistical Analysis. One-way analysis of variance (ANOVA) was used for statistical analysis of the differences between groups. Data were analyzed statistically and have been expressed as mean \pm standard deviation. IBM SPSS Statistics var. 17.0 (IBM Co., Armonk, NY, USA) was used to evaluate data. A value of $P<0.05$ was considered statistically significant.

\section{Results and Discussions}

3.1. Arctiin and Arctigenin Attenuate Silicosis by Inhibiting TLR-4/NLRP3/TGF- $\beta$ Signal Transduction. After rats were exposed to silica for 36 days, the surfaces of lung tissues were uneven locally, exhibiting a hard texture, poor elasticity, and increased volume, with a scattering of small white nodules (Figure Figure S1a). Through H\&E staining, the lesions indicated inflammatory cell infiltration, pulmonary edema, edema in alveolar cavities, widened alveolar septa, increased pulmonary interstitial red blood cells, collagen deposition, and patchy fibrosis, showing a range of observations from mild inflammatory signs to fibrinous exudates and partial fibrous tissue proliferation. Masson's staining of MOD group lung sections showed a large blue area, indicating that the proliferation of collagen fibers was serious and that pulmonary fibrosis occurred (Figures S1b-S1e). Along with time, the appetite of rats in the MOD group became worse compared with the CON group, accompanied with low spirits, sluggish action, lusterless hair, irritability, tachypnea, and weight loss. This situation was in reversed tendency after treatment with arctiin and arctigenin (Figures S2a-S2f).

Serum lysozyme and ceruloplasmin were increased in dust-induced PF [30,31] because lysosomes in cells were damaged and released lysozymes to stimulate fibroblast proliferation after macrophages swallowed the dust [32]. Ceruloplasmin promoted amino oxidation of lysine in peptide chains and eventually resulted in the formation of collagen fibrosis [33]. The level of hydroxyproline could indirectly reflect the degree of fibrosis because it was a unique amino acid in collagen $[34,35]$. Compared with the CON group, the MOD group showed significantly increased levels of hydroxyproline, a specific marker of collagen fiber. The organ coefficient also reflected the reconstruction of lungs (Figures S2g-S2j). Serum ceruloplasmin and lysozyme were low in the CON group, but were increased significantly in the MOD group. After treatment, the activity of ceruloplasmin and the levels of hydroxyproline in the serum recovered to normal in different degree trends (Figures 1(b)-1(d)); particularly, the activity of ceruloplasmin was considerably restored in the AGP group.

Toll-like receptors (TLRs), a type of innate immunity pattern recognition receptor, can be triggered by endogenous danger signals released by macrophages, and the expression of TLR-4 can promote fibrosis formation [36, 37]. The increased expression level of Myd88, the main adaptor protein in the TLR-4 signaling pathway, could drive TAK1 via polyubiquitin chains generated by TRAF6 to activate NF$\kappa \mathrm{B}[38,39]$. In contrast, the NF- $\kappa \mathrm{B}$ signaling pathway can mediate the synthesis of collagen; promote the transcription of cytokines such as TNF- $\alpha$, IL- $1 \beta$, and TGF- $\beta$ [40]; and also induce chronic inflammation by activating the NLRP3 inflammasome [41-44]. The activation of inflammasome is essential for the inflammatory process leading to fibrosis [41]. Numerous inflammatory factors in chronic inflammation can regulate the fibrosis signal transduction cascade and induce fibrosis and malignant tumors [45-48]. However, TGF- $\beta$ plays a central role in fibroblast activation and fibroblast differentiation into myofibroblasts [49-52], contributing to collagen production and extracellular matrix precipitation [53]. $\alpha$-SMA is a myofibroblast-specific protein [54], while the synthesis of collagen is closely linked to hydroxyproline [55]. Compared with the MOD group, the CON group showed significantly low levels of immune cytokines TLR- 4 and Myd88; proinflammatory cytokines TNF- $\alpha$, IL- $1 \beta$, NF- $\kappa \mathrm{B}$, and NF- $\kappa \mathrm{B}$ p 65 ; inflammasome proteins NLRP3, ASC, and cleaved caspase-1; profibrosis cytokine TGF- $\beta$; and fibrosis marker protein $\alpha$-SMA in lung tissues. The increasing protein level in the MOD group indicated that the NLRP3 multiprotein was activated to stimulate the secretion of IL- $1 \beta$ via an immunoinflammatory response, and that the lung fibroblasts transformed into myofibroblasts after silica inhalation. The levels of TNF- $\alpha$, IL- $1 \beta$, NF- $\kappa$ B, TGF- $\beta$, and $\alpha$-SMA in the POS group; TNF- $\alpha$, NF- $\kappa$ B, TLR-4, NLRP3, and $\alpha$-SMA in the ACL group; TNF- $\alpha$, IL- $1 \beta$, NF$\kappa \mathrm{B}$, TGF- $\beta$, TLR- 4 , NF- $\kappa \mathrm{B}$ p 65 , cleaved caspase- 1 , and $\alpha$ SMA in the ACH group; TGF- $\beta$ and TLR- 4 in the AGL group; TGF- $\beta$, ASC, cleaved caspase- 1 , and $\alpha$-SMA in the AGH group; TNF- $\alpha$, NF- $\kappa$ B, TGF- $\beta$, TLR- 4 , Myd88, NF- $\kappa$ B p65, ASC, cleaved caspase- 1 , and $\alpha$-SMA in the ACP group; and TLR-4 and ASC in the AGP group were significantly reduced compared with the levels in the MOD group (Figures 1(e)-1(p)).

3.2. Arctiin and Arctigenin Inhibit Oxidative Stress and Inflammation Induced by Silica in RAW 264.7 Cells. Arctiin and arctigenin showed obvious cytotoxicity at $10 \mu \mathrm{M}$ and no cytotoxicity at $1 \mu \mathrm{M}$ in RAW 264.7 cells exposed to silica (Figure 2(a)). JC-1 aggregates/JC-1 monomers were decreased in the MOD group compared with the CON group, whereas the ARG group was better than the ARC group for their increase (Figures 2(b) and 2(c)). Levels of ROS displayed a contrary tendency, rising in the MOD group but declining much more in the ARG group than in the ARC group (Figures 2(b) and 2(d)). We concluded that silica could induce macrophage 


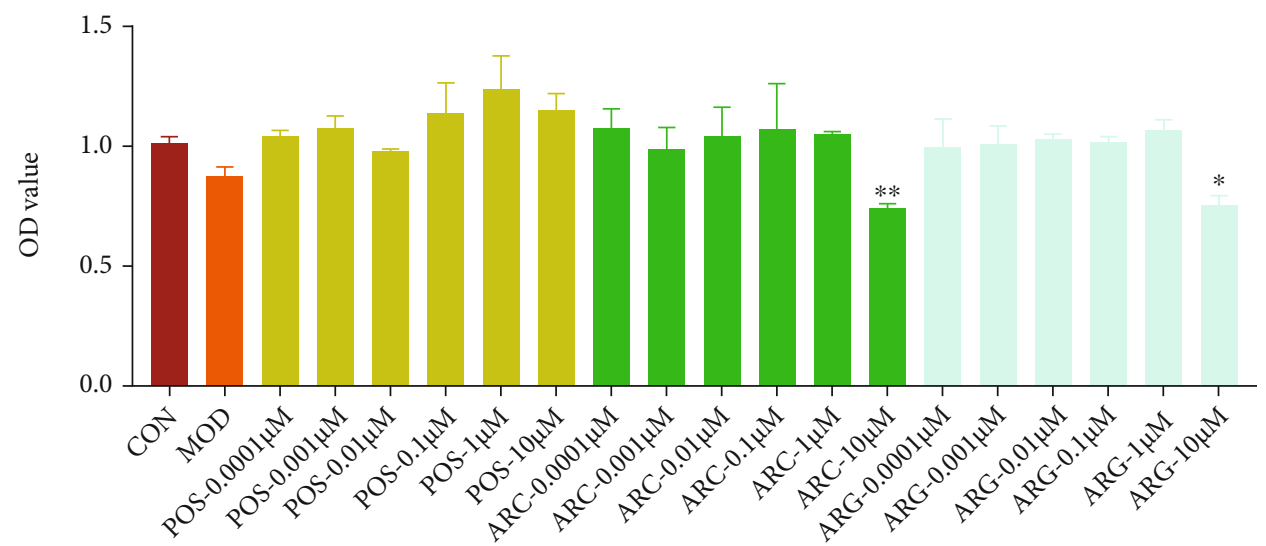

(a)

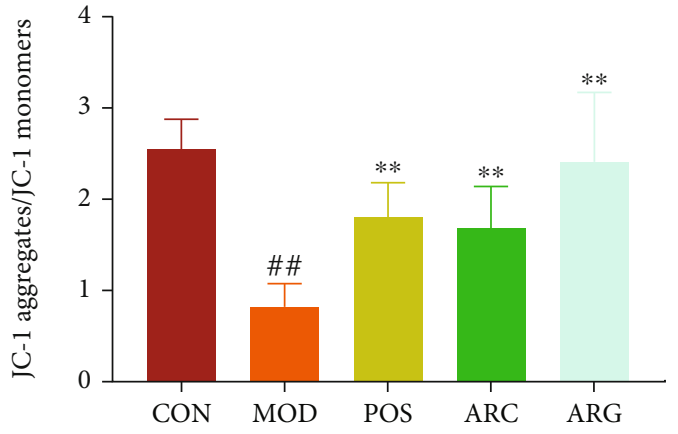

(b)

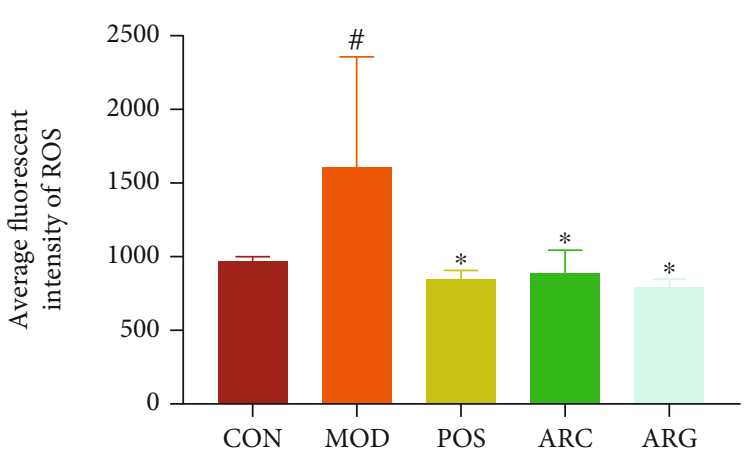

(c)
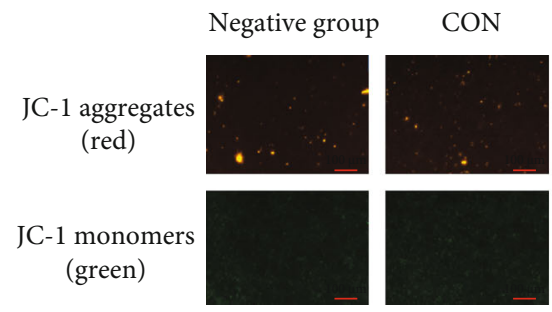

MOD

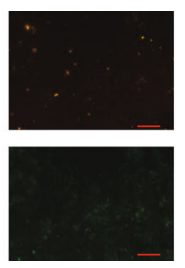

POS

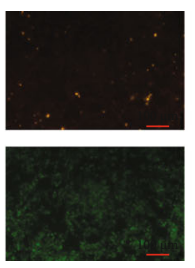

ARC

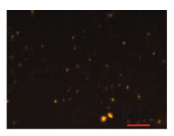

ARG
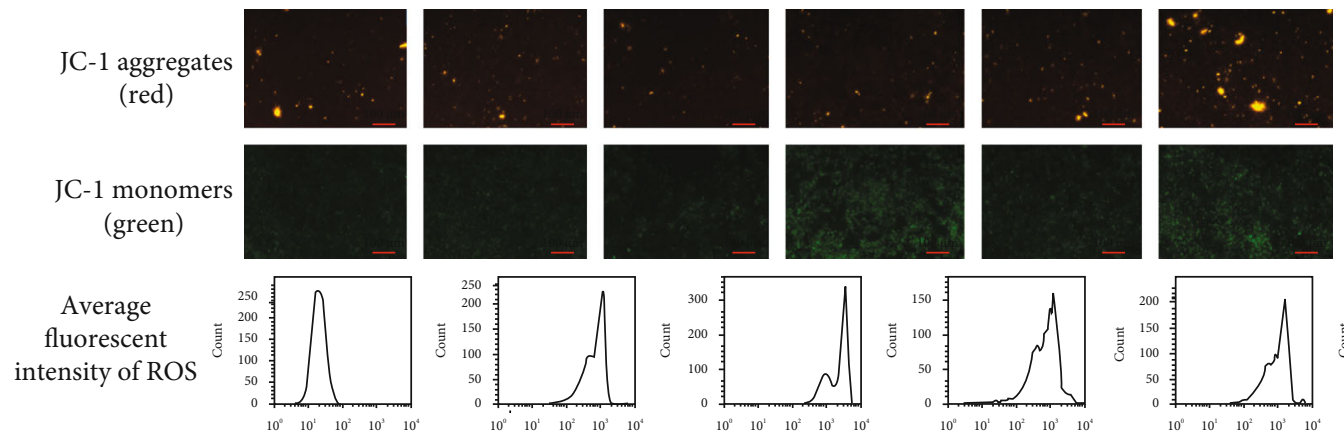

FL1-H

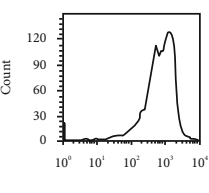

(d)

FIGURE 2: The toxicity of arctiin and arctigenin on macrophages (a). The mitochondrial membrane potential (JC-1 aggregates/JC-1 monomers) and the levels of ROS (b-d). Note: ${ }^{\#} P<0.05$ and ${ }^{\# \#} P<0.01$ compared with CON; ${ }^{*} P<0.05$ and ${ }^{* *} P<0.01$ compared with MOD.

polarization and could increase the expression levels of iNOS and Arg-1, representing that the M0 of macrophages polarized to M1 and M2. Besides, the levels of TLR-4, NLRP3, cleaved caspase-1, and TGF- $\beta 1$ were increased, indicating that cell inflammation occurred on macrophage polarization. Our results indicated that the increased expression levels of iNOS and Arg-1 induced by silica in the ARC group and the ARG group were both downregulated, indicating that the effects of the ARC group and the ARG group on silicosis may be associated with the blocking of the TLR-4/NLRP3/TGF- $\beta$ signaling pathway (Figures $3(\mathrm{a})-3(\mathrm{~g})$ ).
3.3. Arctiin and Arctigenin Inhibit TGF- $\beta 1-I n d u c e d$ Myofibroblast Differentiation. Arctiin showed no obvious cytotoxicity on PLFs at $1 \mu \mathrm{M}$, whereas arctigenin was cytotoxic (Figure 3(h)). Both the ARC group and the ARG group could reduce the expression of $\alpha$-SMA, which was increased by myofibroblast differentiation induced by TGF- $\beta 1$. However, arctiin had a better effect than arctigenin given its lower toxicity (Figures 3(i) and 3(j)).

3.4. Silicosis Progression Based on Metabolomics. Day 7, day 21 , and day 35 were defined as the early, middle, and final phases of silicosis. Differences between the MOD group and 


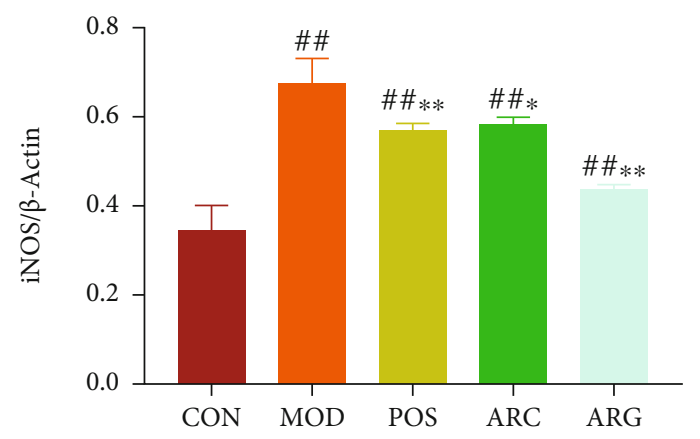

(a)

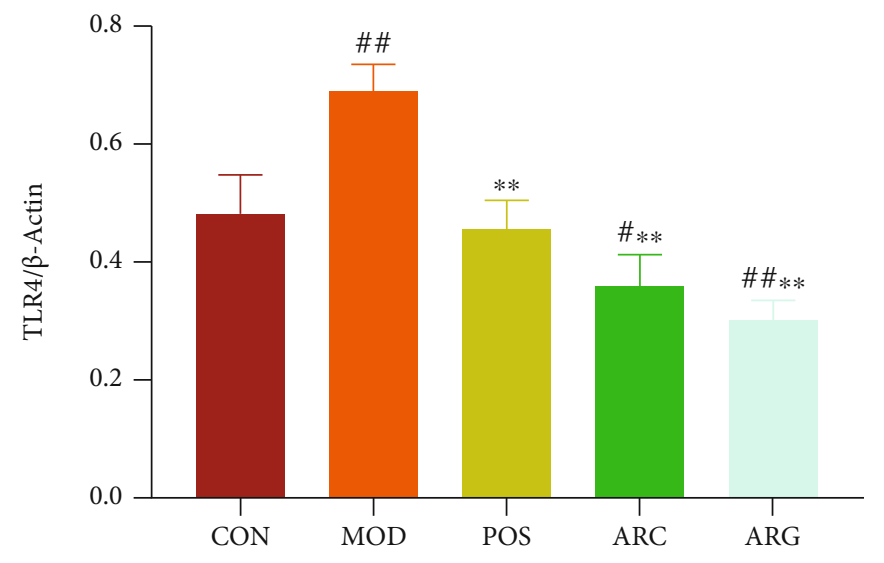

(c)

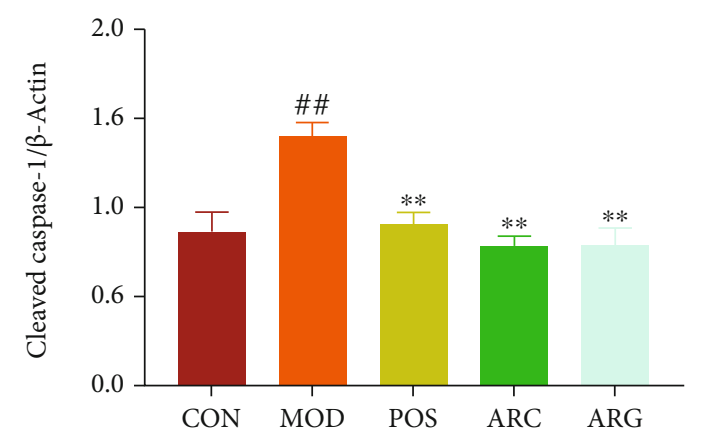

(e)

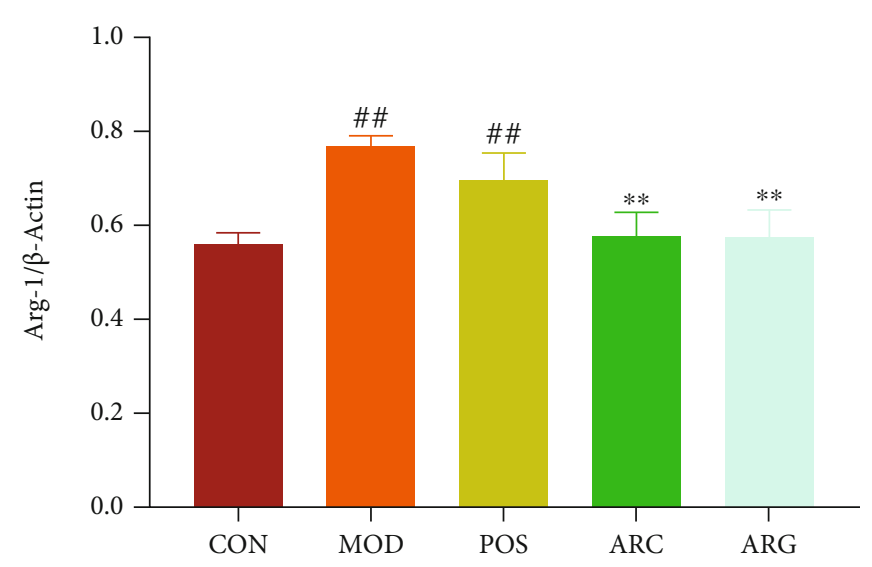

(b)

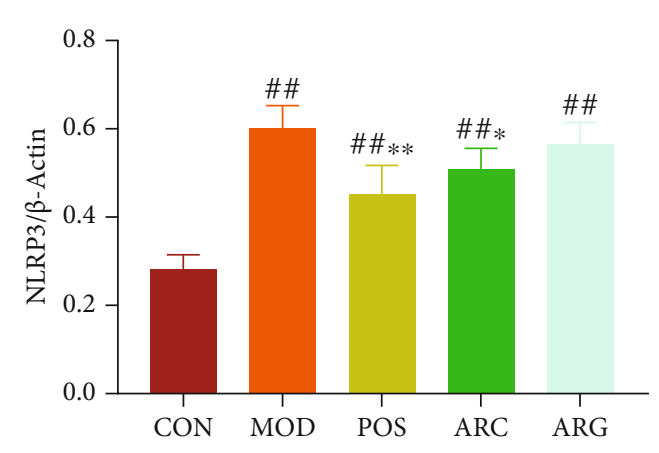

(d)

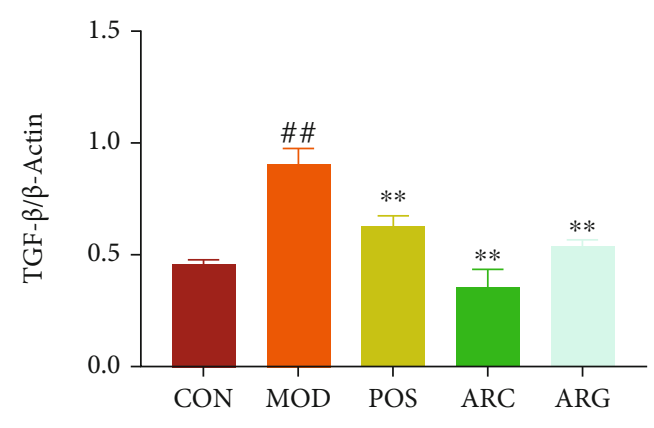

(f)

Figure 3: Continued. 


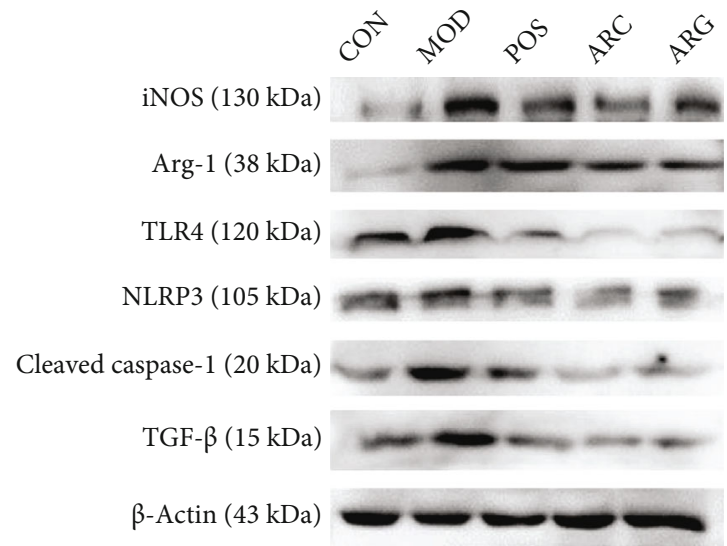

(g)

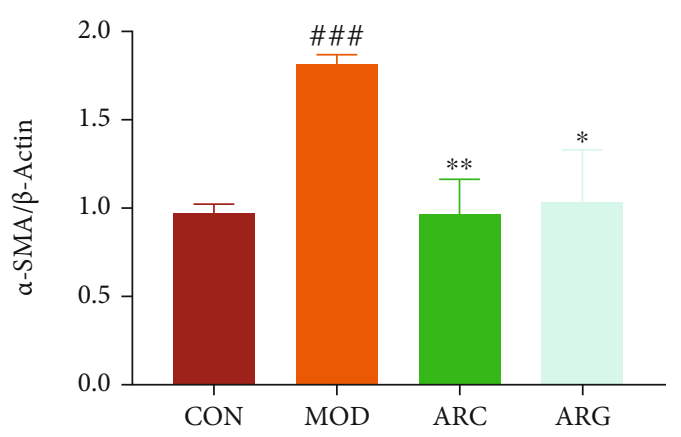

(i)

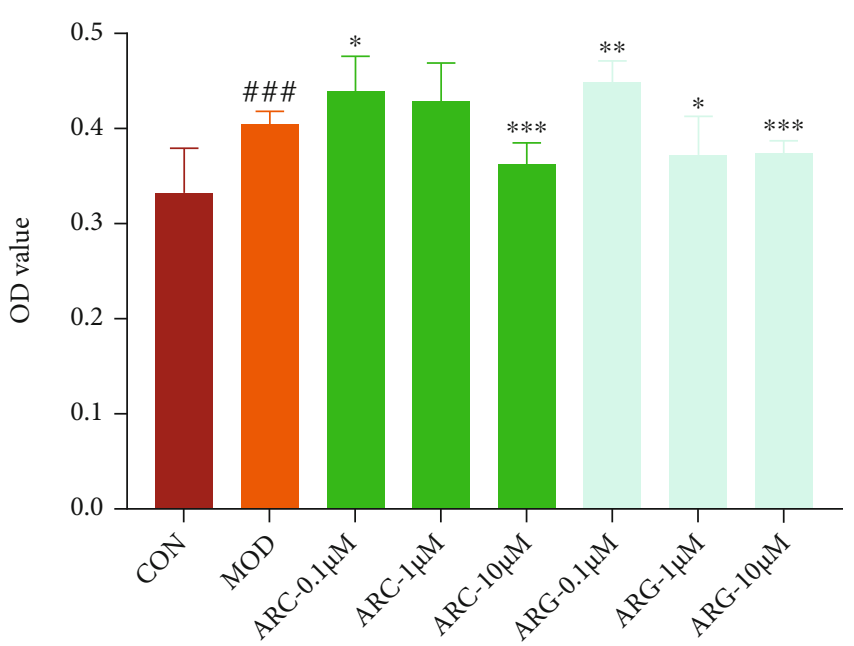

(h)

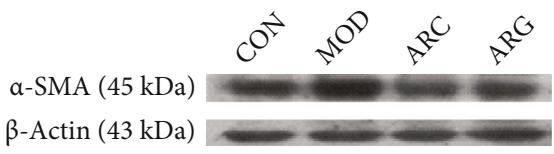

(j)

Figure 3: The expression of iNOS, Arg-1, TLR-4, NLRP3, cleaved caspase-1, and TGF- $\beta$ by Western blot (a-g). The toxicity of arctiin and arctigenin on PFs (h). The expression of $\alpha$-SMA by Western blot $(\mathrm{i}-\mathrm{j})$, mean $\pm \mathrm{SD}, n=3$. Note: ${ }^{\#} P<0.05,{ }^{\# \#} P<0.01$, and ${ }^{\# \# \#} P<0.001$ compared with $\mathrm{CON} ;{ }^{*} P<0.05,{ }^{* *} P<0.01$, and ${ }^{* * *} P<0.001$ compared with MOD.

the CON group increased gradually with the progress of silicosis on OPLS-DA (Figure S3a). In all, 56 metabolites in the early phase, 58 metabolites in the middle phase, and 52 metabolites in the final phase were identified by targeted and untargeted metabolomics. Further, 27, 25, and 33 of these exhibited significant differences (Tables S1-S4).

Metabolic pathways indicated that the early phase of silicosis was associated with pantothenate and CoA biosynthesis, cysteine and methionine metabolism, and taurine and hypotaurine metabolism. The middle phase was closely associated with tryptophan metabolism, pantothenate and CoA biosynthesis, and linoleic acid metabolism among others. The final phase was associated with arginine biosynthesis, butanoate metabolism, and arginine and proline metabolism (Figures 4(a)-4(d)). The metabolic pathway network was established according to the KEGG database, indicating that the mechanism underlying the development of silicosis was closely associated with inflammation, oxidative stress, and lipid and amino acid synthesis and decomposition (Figure 4(e)).

According to the pathway analysis of urine metabolites in various phases of silicosis, we found that in the early and middle phases of silicosis, leukotriene B4 and L-cysteine, which might be associated with inflammation and antioxi- dant mechanisms, were upregulated. The self-protection process of the organism was initiated, and the antioxidant and immune functions were improved to antagonize external stimulation [56]. In the middle phase, upregulated PC and LysoPC may be associated with the destruction of cell membrane structure by silica, causing lipid peroxidation [57]. Arginine and proline metabolism, which promoted hydroxyproline synthesis, was activated, reflecting the presence of collagen synthesis and the occurrence of fibrosis $[58,59]$.

Based on the abovementioned research results of metabolomics and signal transduction, we found that oxidative stress, inflammation, and enhanced innate immune responses were the main reactions after silica was phagocytosed by macrophages in the early phase. NLRP3 inflammasome was activated through the TLR$4 / \mathrm{Myd} 88 / \mathrm{NF}-\kappa \mathrm{B}$ cascade, promoting the secretion of inflammatory factors IL- $1 \beta$ and TNF- $\alpha$ and the fibrogenic factor TGF- $\beta$. Substance metabolism was guided and regulated through signal transduction, and signal molecules activated a series of cascade reactions by binding with receptors to transmit external information to intracellular regions, affecting substance metabolism, gene expression, or biological functioning of cells. Metabolomics indicated that arginine and proline metabolism made a considerable 


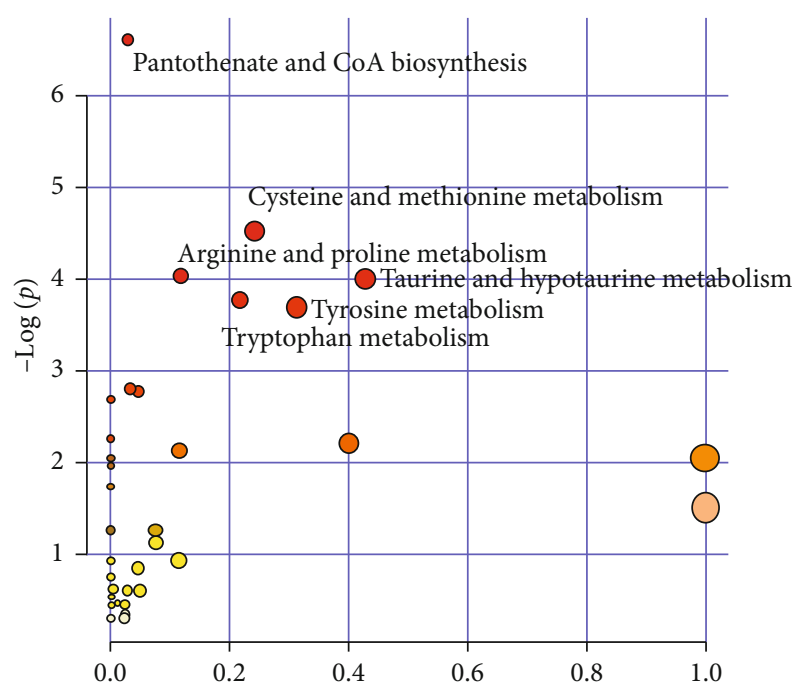

Pathway impact

(a)

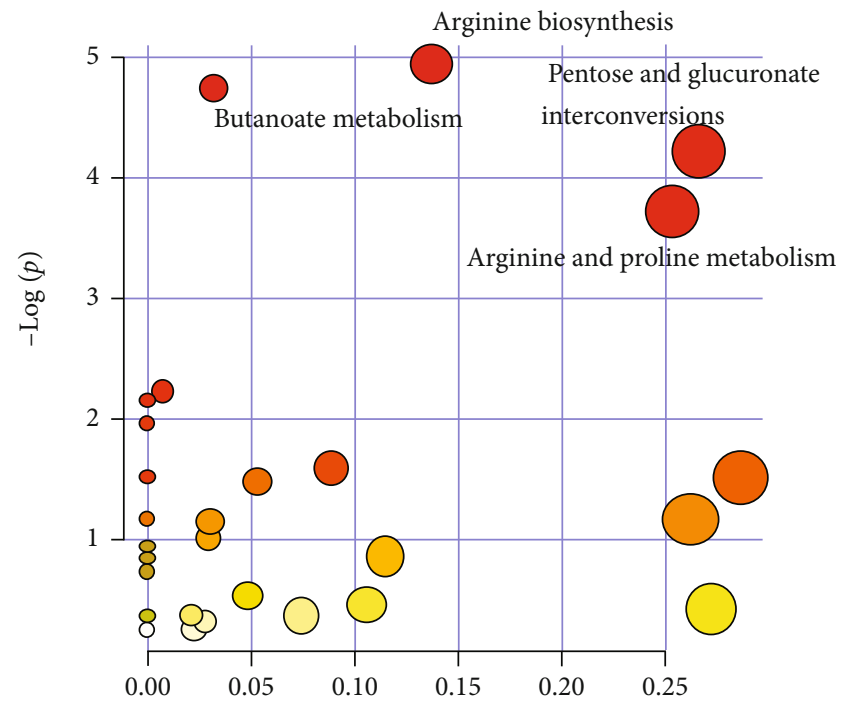

Pathway impact

(c)

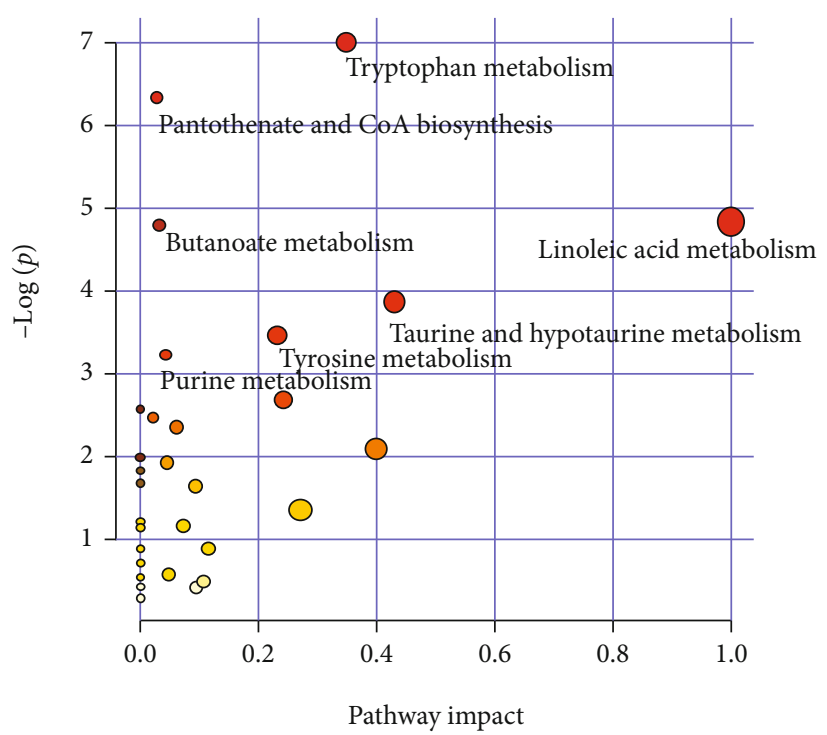

(b)

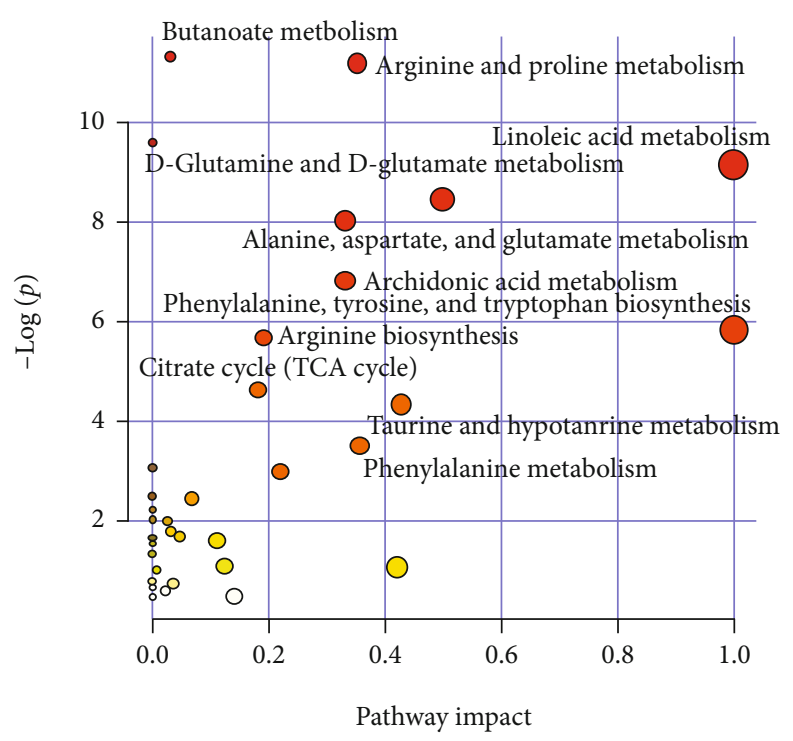

(d)

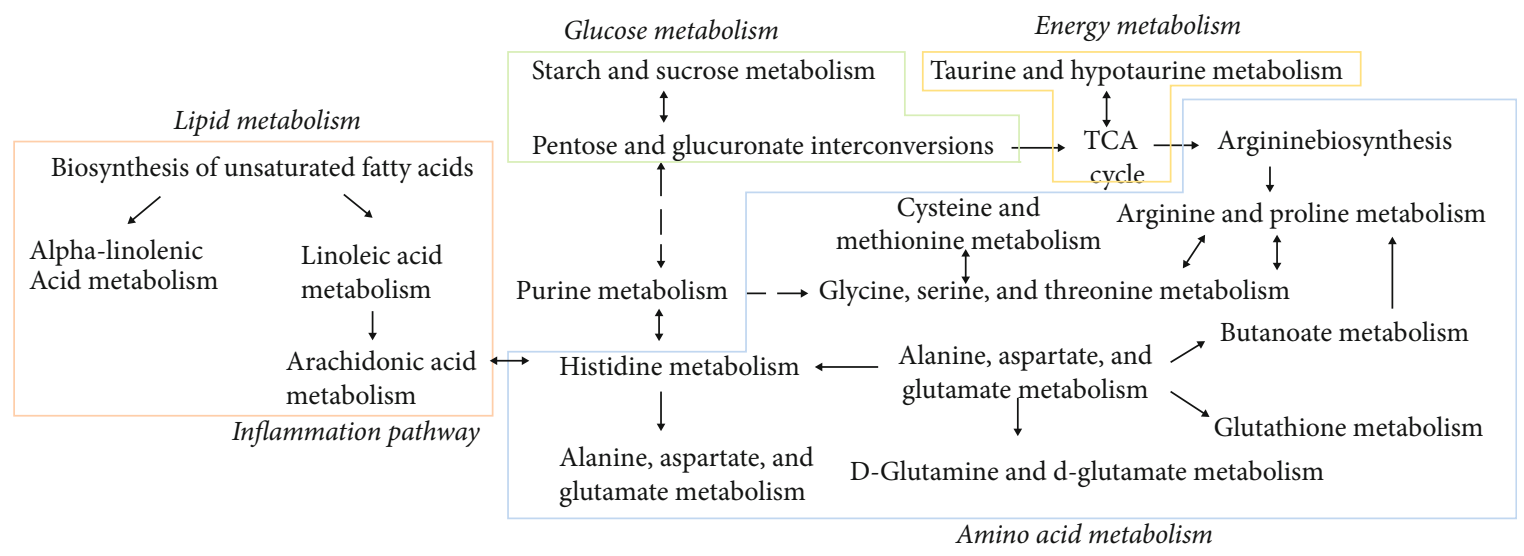

(e)

FIGURE 4: Metabolic pathway analysis by MetaboAnalyst: day 7 urine (a), day 21 urine (b), day 35 urine (c), day 36 serum (d), and metabolic pathway network (e). 

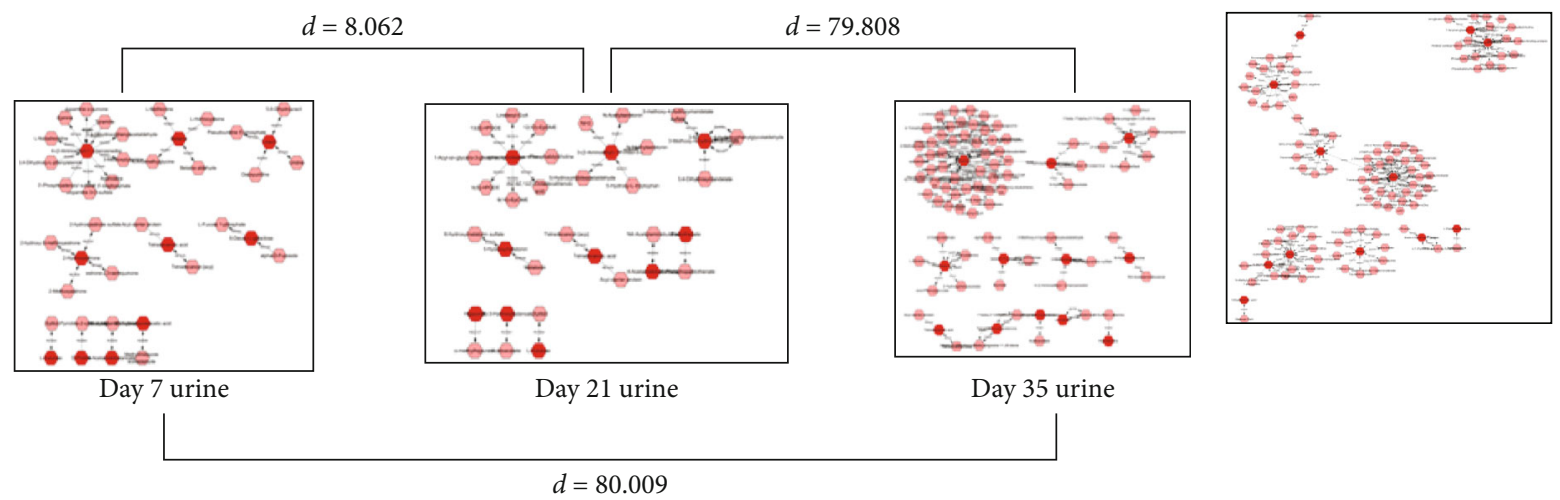

(a)

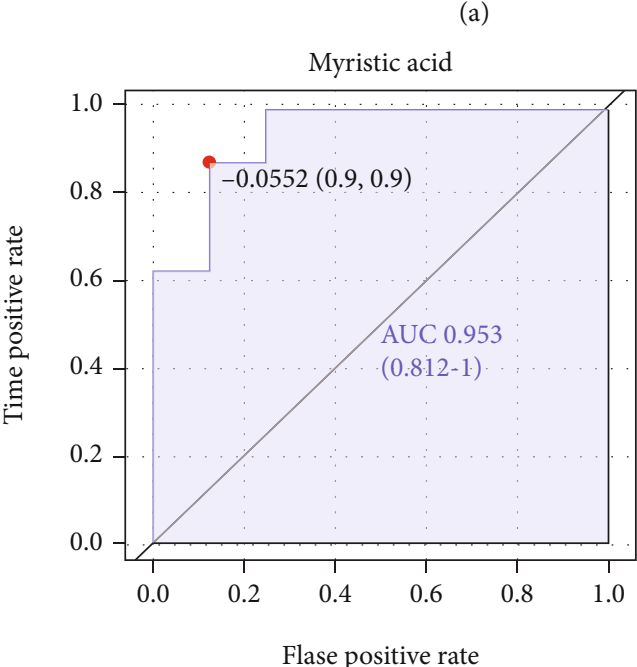

(c)

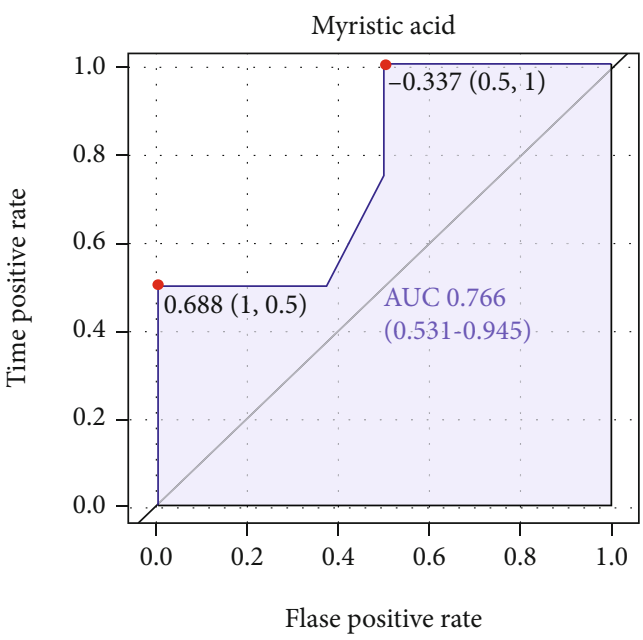

(e)

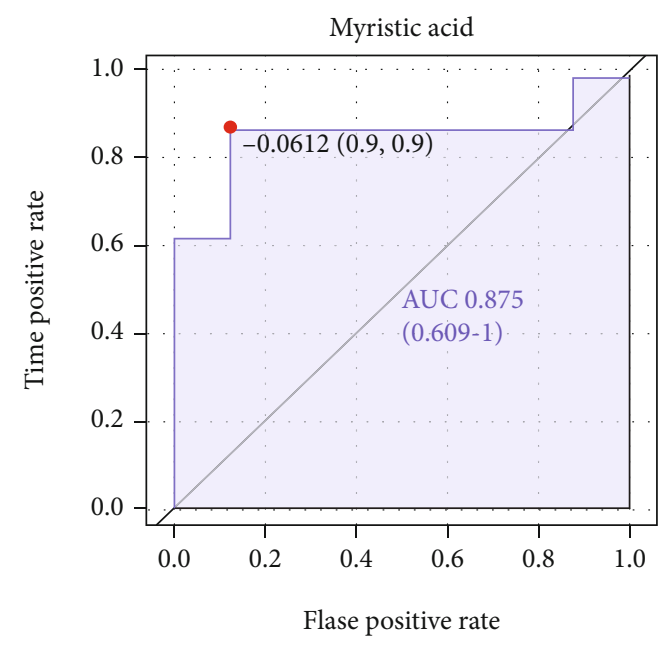

(d)

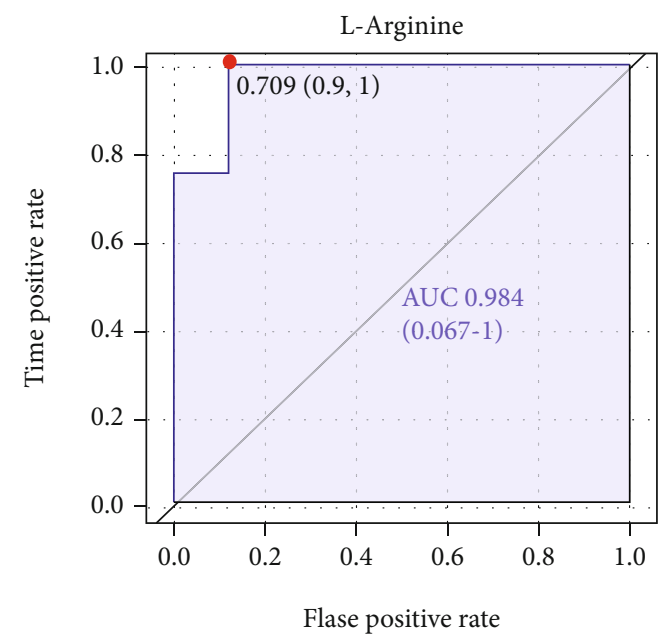

(f)

FIGURE 5: Continued. 


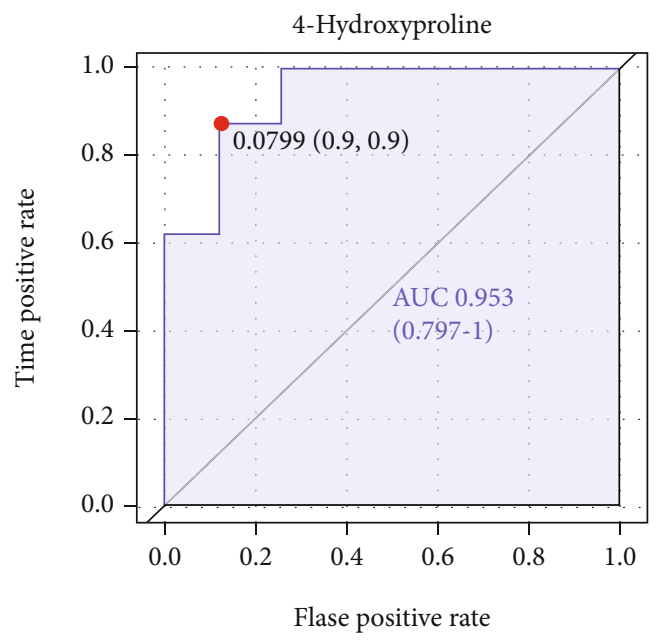

(g)

Figure 5: Metabolic network and the prediction of diagnostic indexes. Topological analyses of urine metabolic network (a) and serum metabolic network (b). ROC analysis of myristic acid, L-arginine, and 4-hydroxyproline (c-g).

contribution to the synthesis of collagen. Therefore, gene PPI interaction analysis of arginine and proline metabolism and TLR-4/NLRP3/TGF- $\beta$ signal transduction was investigated to elucidate the relationship between substance metabolism and signal transduction. Figure S4 shows that there are six related genes between TLR4/NLRP3/TGF- $\beta$ signal transduction and arginine and proline metabolism, including ARG1, ARG2, NOS1, NOS2, NOS3, and ALDH7A1. NOS2 (iNOS) and Arg-1 were macrophage polarization markers of M1 and M2 macrophages, respectively. The NLRP3 inflammasome could mediate M1 macrophage polarization to promote the production of IL- $1 \beta$ and upregulate the expression of IL-4 to stimulate M2 macrophage polarization [60-62]. Arctiin and arctigenin could both inhibit macrophage M0 polarization to M1, alleviate proinflammatory response, and reduce the release of inflammatory factors. The polarization of M1 to M2 was also blocked; this may be because of a decrease in the level of M1 macrophages. The blockage of macrophage polarization may be associated with the blocking of TLR4/NLRP3/TGF- $\beta$ signaling.

3.5. Topological Analysis of Metabolic Network in Different Phases and the Prediction of Diagnostic Indices of Silicosis. The metabolic networks in different periods of silicosis are displayed in Figures 5(a) and 5(b). Urine metabolic network topology parameters (Table S5) were used to calculate the $d$ value, representing dissimilarity, according to the Euclidean Distance Formula. This means that the smaller the $d$ values are, the more similar the networks are. Figure 5(a) shows that the similarity between the early and final phases was the largest; the similarity between the early and middle phases was smaller than that between the early and final phases. After the overall evaluation of different phases, the degree and betweenness centrality were used to further investigate the importance of nodes in different phases. In this study, myristic acid in the urine was selected as a critical metabolite, L-arginine and 4-hydroxyproline in serum were also prominent in the network (Table S6).

11 metabolites were coexisting in urine during different periods in the MOD group compared with the CON group. The levels of myristic acid, uracil, mevalonic acid, 3hydroxybutyric acid, pantothenic acid, epinephrine, and succinic acid semialdehyde were downregulated, whereas those of 3,6-octadienoylglycine, 1-methyluric acid, 4-hydroxyproline, and 1-methylhistidine were upregulated in a timedependent manner (Table 1). Myristic acid, 3,6-octadienoylglycine, and uracil in the early phase; myristic acid, 3,6-octadienoylglycine, 3-hydroxybutyric acid, and pantothenic acid in the middle phase; and myristic acid, 3,6-octadienoylglycine, 3-hydroxybutyric acid, and 1-methyluric acid in the final phase satisfied the differential metabolite requirements for VIP $>1$ and $P<0.05$ in metabolomics. Subsequently, ROC analysis was conducted, and the diagnostic value of metabolites was evaluated with the AUC cut-off of 0.9. The AUC of myristic acid was up to 0.953 , indicating a high diagnosis value in the early phase, and it reduced to 0.875 and even 0.766 gradually with the development of silicosis (Figures 5(c)-5(e)). Besides, the AUCs of serum L-arginine and 4-hydroxyproline were also high, at 0.984 and 0.953 respectively (Figures $5(\mathrm{f})$ and $5(\mathrm{~g})$ ), and they may be effectively used as biomarkers for pulmonary fibrosis.

Myristic acid is a type of saturated fatty acid [63]. Proteins can acylate through the $\mathrm{N}$-terminal myristoylation reaction of myristic acid, which has a membrane localization effect. This membrane localization effect can promote NO release from the cells, and the oxidative stress of NO can help macrophages against the pathogens in the immune system. However, unmyristoylated proteins bind to the receptor CD36 and then activate NOS by influencing AMP kinase through Src kinase [64]. The importance of NOS in arginine and proline metabolism also reflects the indirect contribution of myristic acid to arginine and proline metabolism. Further, the content of hydroxyproline, which is a unique amino acid in collagen fibrosis, can reflect the degree of fibrosis [65]. The 
TABLE 1: The mutual metabolites in urine of various periods.

\begin{tabular}{|c|c|c|c|c|c|c|}
\hline \multirow{2}{*}{ Metabolites } & \multicolumn{2}{|c|}{ Day 7} & \multicolumn{2}{|c|}{ Day 21} & \multicolumn{2}{|c|}{ Day 35} \\
\hline & Trend & VIP & Trend & VIP & Trend & VIP \\
\hline Myristic acid & +++ & 3.461 & ++ & 1.509 & ++ & 2.133 \\
\hline 3,6-Octadienoylglycine & ++ & 2.105 & - & 1.946 & ++ & 1.828 \\
\hline Uracil & ++ & 1.968 & l & 0.063 & + & 1.161 \\
\hline Mevalonic acid & + & 1.663 & l & 0.021 & l & 0.189 \\
\hline 3-Hydroxybutyric acid & - & 1.078 & - & 1.501 & - & 1.943 \\
\hline Pantothenic acid & + & 0.824 & ++ & 1.409 & l & 0.528 \\
\hline 1-Methyluric acid & - & 0.822 & l & 0.092 & ++ & 1.723 \\
\hline Epinephrine & + & 0.578 & + & 0.924 & l & 0.055 \\
\hline 4-Hydroxyproline & l & 0.299 & + & 1.119 & + & 1.106 \\
\hline Succinic acid semialdehyde & l & 0.252 & - & 0.241 & - & 0.327 \\
\hline 1-Methylhistidine & - & 0.213 & l & 0.004 & + & 1.148 \\
\hline
\end{tabular}

Note: “-” represents metabolites with a downregulated trend, “+” represents metabolites with an upregulated trend, “-” and “++” represent significant difference from control at $P<0.05$, and “-” and " +++ " represent significant difference from control at $P<0.01$.

level of urine hydroxyproline in the early phase of silicosis showed no difference when compared with the level in the CON group, representing that there was no fibrosis during that time, whereas upregulating trends were observed in the middle and final phases of silicosis. Particularly, significantly different levels were observed in the serum, representing fibrosis induced by the formation of silica. Initially, fibrosis was evaluated by hydroxyproline alone. This study further revealed that the dynamic changes in myristic acid or 4hydroxyproline could reflect the degree of fibrosis: myristic acid was downregulated and 4-hydroxyproline was upregulated with the progression of silicosis. However, the effect of arctigenin on myristic acid was not as good as that of arctiin in the final phase of silicosis, mostly because of the toxicity of arctigenin as a result of in vivo accumulation with long-term administration [66, 67]. Results of the MTT assay of cells also reflected that the toxicity of arctigenin was higher than that of arctiin at the same concentration. Besides, neither arctiin nor arctigenin showed significant regulatory effects on L-arginine, but both arctiin and arctigenin could downregulate 4-hydroxyproline compared with the levels in the MOD group. L-Arginine and 4-hydroxyproline both participated in arginine and proline metabolic pathways, but L-arginine was not involved in the synthesis of collagen.

3.6. Effects of Arctiin and Arctigenin on the Different Phases of Silicosis by Metabolomics. The metabolic networks are displayed in Figure 6. OPLS-DA results displayed as score plots could indicate the scatter of the samples, wherein similar metabolomic compositions were clustered together and compositionally different metabolomic compositions were dispersed. The score plots of urine showed that the ACL group and the ACH group were more similar to the CON group, whereas the AGL group and the AGH group were closer to the MOD group, indicating that the ACL group and the $\mathrm{ACH}$ group could regulate metabolic disorders effectively. The OPLS-DA results of serum metabolism showed a similar trend (Figure S3b).
Based on the abovementioned results, myristic acid in urine had a higher diagnostic value in the early phase, and L-arginine and 4-hydroxyproline in the serum had higher diagnostic values in the final phase of silicosis. Therefore, the regulatory effects of arctiin and arctigenin on these high-value metabolites could evaluate their efficacy. The results showed that arctiin and arctigenin could both modify myristic acid levels in the early phase with similar strength, and arctigenin had a better effect on myristic acid in the middle phase than arctiin, whereas arctiin had a better effect than arctigenin in the final phase of silicosis. Further, the efficacy of a combined medication with arctiin and positive drugs was better than that of arctiin alone, showing a synergistic effect. There was no obvious regulatory effect of arctiin and arctigenin on L-arginine in the serum, but the efficacy was increased with combined medication using arctiin and positive drugs. Arctiin had a better effect on serum 4hydroxyproline than arctigenin (Tables S1-S4).

Compared with the MOD group, the arctiin groups showed significant differences in 11 of 56 urine metabolites in the early phase, 8 of 58 urine metabolites in the middle phase, and 15 of 52 urine metabolites and 16 of 46 serum metabolites in the final phase. In contrast, there were 16 of 56 urine metabolites in the early phase, 11 of 58 urine metabolites in the middle phase, and 22 of 52 urine metabolites and 24 of 46 serum metabolites in the final phase that showed significant differences after treatment with arctigenin. It can be clearly concluded that arctigenin regulated more metabolites than arctiin. The pathways of these abovementioned metabolites indicated that arctiin could regulate the biosynthesis of hormones in the early phase; taurine and hypotaurine metabolism in the middle phase; and taurine and hypotaurine metabolism, arginine and proline metabolism, glycerophospholipid metabolism, and phenylalanine, tyrosine, and tryptophan biosynthesis in the final phase of silicosis, whereas arctigenin could regulate arginine and proline metabolism and synthesis and degradation of ketone bodies in the early phase; linoleic acid metabolism in the middle phase; and linoleic acid metabolism, pantothenate and CoA 


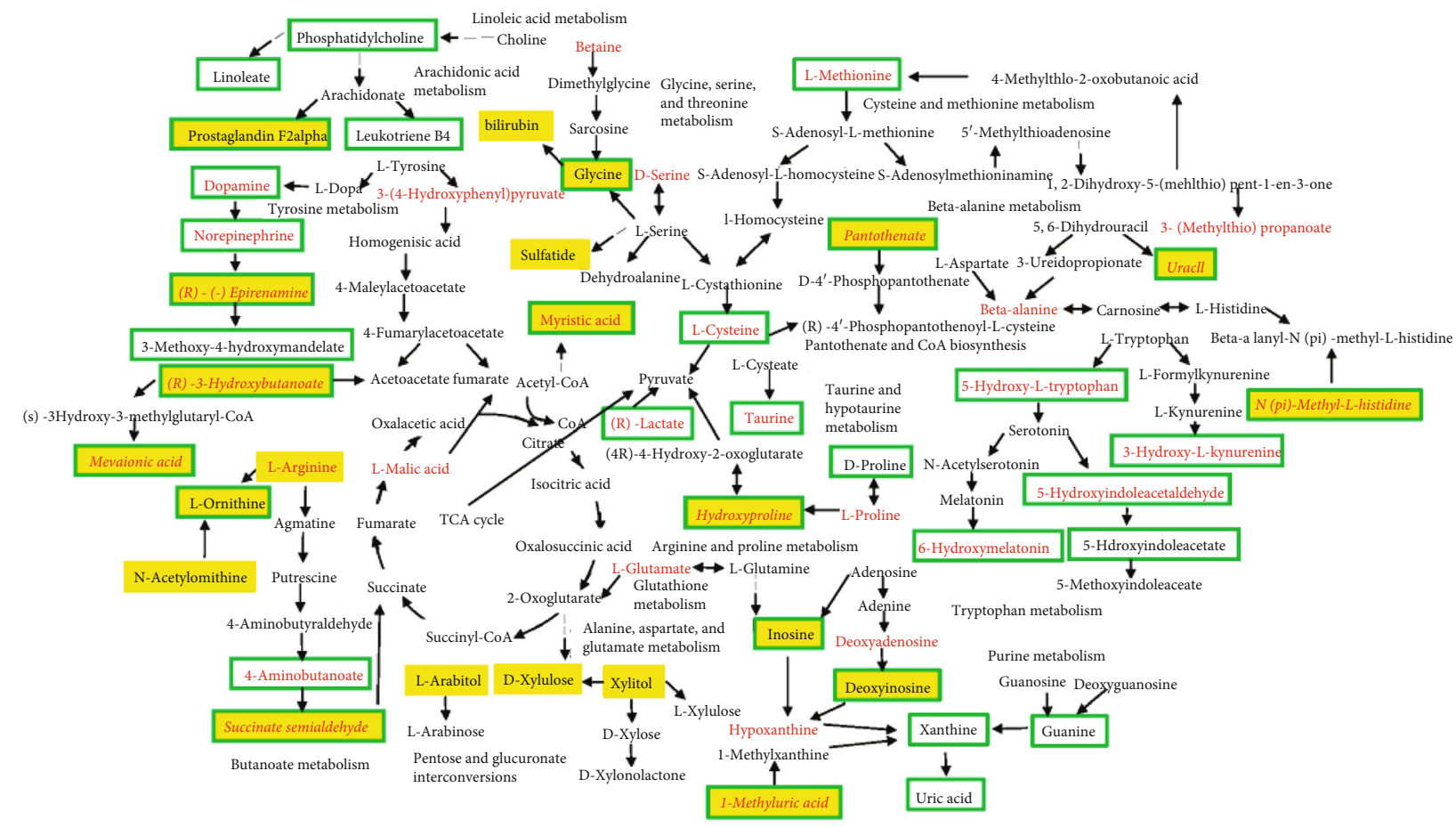

FIGURE 6: Urine metabolic network (note: early metabolites in red, middle metabolites in yellow, and final metabolites in green).

biosynthesis, $\beta$-alanine metabolism, arachidonic acid metabolism, butanoate metabolism, arginine and proline metabolism, alanine, aspartic acid and glutamic acid metabolism, and the metabolism of glycine, serine, and threonine in the final phase of silicosis (Tables S1-S4). Above all, arctiin and arctigenin could regulate energy metabolism and glucose metabolism by intervening in lipid metabolism and inflammation-related pathways and inhibit collagen synthesis to attenuate silicosis.

3.7. Network Pharmacological Analysis to Determine the Regulatory Effect of Arctiin and Its Metabolites on Silicosis. The network pharmacology of arctiin and its 15 metabolites was performed, and pathway enrichment results of network pharmacology showed the TNF signaling pathway, the NOD-like receptor signaling pathway, Toll-like receptor signaling, the MAPK signaling pathway, and 90 related pathways, indicating that arctiin and its metabolites play important roles in the treatment of silicosis. Biological function analysis further indicated that the regulatory effect of arctiin and its metabolites on silicosis was associated with biological processes such as the lipopolysaccharidemediated signaling pathway; positive regulation of the ERK1 and ERK2 cascade; cellular response to mechanical stimulus; and molecular functions such as interleukin-1 receptor binding, MAP kinase activity, and ATP binding (Figure S5), providing a new direction for the development of drugs. Research indicated that ATP, which might be relied on energy metabolism or glucose metabolism, was needed for the activation of the NLRP3 inflammasome [68].

3.8. Oral Bioavailability Prediction and Molecular Docking Studies of Arctigenin and Its Metabolites. Arctiin and arcti- genin could inhibit the conversion of fibroblasts to myofibroblasts and reduce the accumulation of extracellular matrix in vitro; however, arctiin was better than arctigenin for the treatment of silicosis being associated with the toxicity of arctigenin. Arctigenin was mostly regarded as the direct effective compound of arctiin in Fructus Arctii, but its oral bioavailability is lower because of its lignan structure and insolubility in water, and it is usually administered in the form of an injection [69]. Arctiin is present in a much higher concentration than arctigenin in Fructus Arctii [70]. Our results indicate that arctiin can be hydrolyzed into arctigenin and has various pharmacological effects given its high polarity and easy oral absorption [71, 72]. The prediction of oral bioavailability of arctiin was $75 \%$, whereas that of arctigenin was $58 \%$ according to AdmetSAR2.0 web-service (Table S7), indicating that arctiin is easier as a medicine because of its high oral bioavailability. Besides, arctiin and its metabolite $4^{\prime}, 4^{\prime \prime}$ -dihydroxyl-enterolactone showed better binding with TGF$\beta \mathrm{RI}$ according to the XP Gscore in molecular docking studies (Table S8). A previous study on the pharmacokinetics of arctiin after oral administration indicated that it had a rapid absorption phase followed by a sharp but lasting disappearance, which indicates that it could be metabolized into arctigenin and other metabolites [28]. Thus, arctiin coexists with lower concentrations of its metabolites being produced by metabolizing arctiin constantly in the organism after oral administration. Compared with the toxicity of arctigenin alone on oral administration, that of arctiin was lower because it constantly produced low concentrations of arctigenin. Thus, arctiin showed a much higher druggability than arctigenin because of its high oral bioavailability and low toxicity. 


\section{Conclusions}

To the best of our knowledge, this is the first study identifying urine myristic acid and serum L-arginine and 4hydroxyproline as biomarkers for silicosis diagnosis. The mechanism underlying the development of silicosis is closely associated with inflammation, oxidative stress, and lipid and amino acid synthesis and degradation. Arctiin and arctigenin could ameliorate oxidative stress, immune-related inflammatory reaction, and fibrosis through pantothenate and CoA biosynthesis, cysteine and methionine metabolism, taurine and hypotaurine metabolism, linoleic acid metabolism, and arginine and proline metabolism successively. The importance of macrophage polarization in fibrosis was verified by integrated arginine and proline metabolism and the TLR4/NLRP3/TGF- $\beta$ signaling pathway, and arctiin and arctigenin could both attenuate the development of silicosis and protect the lungs from injury by blocking the polarization of macrophages and inhibiting the differentiation of myofibroblasts by regulating TLR-4/NLRP3/TGF- $\beta$ signal transduction. Our research for the first time reveals that both arctiin and arctigenin are effective in silicosis, with the former showing better druggability.

\section{Data Availability}

The metabolomics data and molecular docking data used to support the findings of this study are included within the supplementary information file, and other data used to support the findings of this study are available from the corresponding author upon request.

\section{Conflicts of Interest}

The authors declare that there is no conflict of interest regarding the publication of this paper.

\section{Acknowledgments}

This work received the support of the National Natural Science Foundation of China (81773858) and the Major Scientific Research Projects of Intercollegiate Cooperation in Colleges and Universities of Liaoning Province (2020), No. 28.

\section{Supplementary Materials}

More details and procedures including materials, dose selection, modeling methods, metabolomics, and cell cultures and some figures and tables to support the results and conclusion are shown in Supplemental materials. (Supplementary Materials)

\section{References}

[1] B. T. Mossman and A. Churg, "Mechanisms in the pathogenesis of asbestosis and silicosis," American Journal of Respiratory \& Critical Care Medicine, vol. 157, 5 Part 1, p. 1666, 2012.

[2] M. Ding, F. Chen, X. Shi, B. Yucesoy, B. Mossman, and V. Vallyathan, "Diseases caused by silica: mechanisms of injury and disease development," International Immunopharmacology, vol. 2, no. 2-3, pp. 173-182, 2002.

[3] O. Takemi, M. Megumi, M. Shuko, H. Hiroaki, and M. Yoshie, "Immunological effects of silica and asbestos," Cellular \& Molecular Immunology, vol. 4, no. 4, pp. 261-268, 2007.

[4] T. Eleftheriadis, G. Pissas, S. Zarogiannis, V. Liakopoulos, and I. Stefanidis, "Crystalline silica activates the T-cell and the Bcell antigen receptor complexes and induces T-cell and B-cell proliferation," Autoimmunity, vol. 52, no. 3, pp. 136-143, 2019.

[5] B. Fubini and A. Hubbard, "Reactive oxygen species (ROS) and reactive nitrogen species (RNS) generation by silica in inflammation and fibrosis," Free Radical Biology and Medicine, vol. 34, no. 12, pp. 1507-1516, 2003.

[6] N. W. Todd, I. G. Luzina, and S. P. Atamas, "Molecular and cellular mechanisms of pulmonary fibrosis," Fibrogenesis \& Tissue Repair, vol. 5, no. 1, 2012.

[7] N. Sakai and A. M. Tager, "Fibrosis of two: epithelial cellfibroblast interactions in pulmonary fibrosis," Biochimica Et Biophysica Acta, vol. 1832, no. 7, pp. 911-921, 2013.

[8] E. J. Want, I. D. Wilson, H. Gika et al., "Global metabolic profiling procedures for urine using UPLC-MS," Nature Protocols, vol. 5, no. 6, pp. 1005-1018, 2010.

[9] R. H. Barton, J. K. Nicholson, P. Elliott, and E. Holmes, "Highthroughput $1 \mathrm{H}$ NMR-based metabolic analysis of human serum and urine for large-scale epidemiological studies: validation study," International Journal of Epidemiology, vol. 37, Supplement 1, pp. i31-i40, 2008.

[10] M. Lauridsen, S. H. Hansen, J. W. Jaroszewski, and C. Cornett, "Human urine as test material in $1 \mathrm{H}$ NMR-based metabonomics: recommendations for sample preparation and storage," Analytical Chemistry, vol. 79, no. 3, pp. 1181-1186, 2007.

[11] C. Si, W. Si, W. Li et al., "Investigation of the therapeutic effectiveness of active components in Sini decoction by a comprehensive GC/LC-MS based metabolomics and network pharmacology approaches," Molecular BioSystems, vol. 10, no. 12, pp. 3310-3321, 2014.

[12] A. Zhang, H. Sun, B. Yang, and X. Wang, "Predicting new molecular targets for rhein using network pharmacology," BMC Systems Biology, vol. 6, no. 1, p. 20, 2012.

[13] National Pharmacopoeia Committee, Pharmacopoeia of the People's Republic of China, Part 1., China Medical Science Press, Beijing, 2020.

[14] X. Kou, S. Qi, W. Dai, L. Luo, and Z. Yin, “Arctigenin inhibits lipopolysaccharide-induced iNOS expression in RAW264.7 cells through suppressing JAK-STAT signal pathway," International Immunopharmacology, vol. 11, no. 8, pp. 11021102, 2011.

[15] W.-z. Zhang, Z.-k. Jiang, B.-x. He, and X.-b. Liu, “Arctigenin protects against lipopolysaccharide-induced pulmonary oxidative stress and inflammation in a mouse model via suppression of MAPK, HO-1, and iNOS signaling," Inflammation, vol. 38, no. 4, pp. 1406-1414, 2015.

[16] G. R. Chen, H. F. Li, D. Q. Dou et al., "(-)-Arctigenin as a lead compound for anticancer agent," Natural Product Research, vol. 27, no. 23, pp. 2251-2255, 2013.

[17] D. Li, Q. Liu, J. Dong, D. Dou, X. Wang, and T. Kang, "Protective effect of arctigenin against MPP+ and MPTP-induced neurotoxicity," Planta Medica, vol. 80, no. 1, pp. 48-55, 2014.

[18] N. Zhang, D. Dou, X. Ran, and T. Kang, "Neuroprotective effect of arctigenin against neuroinflammation and oxidative 
stress induced by rotenone," RSC Advances, vol. 8, no. 5, pp. 2280-2292, 2018.

[19] A. Li, J. Wang, D. Zhu, X. Zhang, R. Pan, and R. Wang, “Arctigenin suppresses transforming growth factor- $\beta 1$-induced expression of monocyte chemoattractant protein- 1 and the subsequent epithelial-mesenchymal transition through reactive oxygen species-dependent ERK/NF- $\kappa \mathrm{B}$ signaling pathway in renal tubular epithelial cells," Free Radical Research, vol. 49, no. 9, pp. 1095-1113, 2015.

[20] F. Gao, Y. Zhang, Z. Yang et al., "Arctigenin suppressed epithelial-mesenchymal transition through Wnt3a/ $\beta$-catenin pathway in PQ-induced pulmonary fibrosis," Frontiers in Pharmacology, vol. 11, article 584098, 2020.

[21] Y. Xu, Z. Lou, and S. H. Lee, “Arctigenin represses TGF- $\beta$ induced epithelial mesenchymal transition in human lung cancer cells," Biochemical \& Biophysical Research Communications, vol. 493, no. 2, pp. 934-939, 2017.

[22] B. Zhou, G. Weng, Z. Huang, T. Liu, and F. Dai, “Arctiin prevents LPS-induced acute lung injury via inhibition of PI3K/AKT signaling pathway in mice," Inflammation, vol. 6, no. 41, pp. 2129-2135, 2018.

[23] B. Min, P. Shin, S. Chun, and J. Chung, “Arctiin regulates lipid metabolism via AMP-activated protein kinase signaling pathways in 3T3-L1 adipocytes," In KFN International Symposium and Annual Meeting, pp. 338-339, 2013.

[24] S. Ma, D.-l. Liu, J.-j. Deng, R. Niu, and R.-b. Liu, "Effect of arctiin on glomerular filtration barrier damage in STZ-induced diabetic nephropathy rats," Phytotherapy Research, vol. 27, no. 10, pp. 1474-1480, 2013.

[25] Y. An, "Effect of arctiin on glucose and lipid metabolism in diabetic mice," Henan Traditional Chinese Medicine, vol. 33, no. 2, pp. 193-195, 2013.

[26] L. C. Lu, W. Zhou, Z. H. Li et al., "Effects of arctiin on streptozotocin-induced diabetic retinopathy in SpragueDawley rats," Planta Medica, vol. 78, no. 12, pp. 1317-1323, 2012.

[27] F. He, D. De-Qiang, S. Yu, L. Zhu, X. Hong-Bin, and K. TingGuo, "Plasma pharmacokinetics and tissue distribution of arctiin and its main metabolite in rats by HPLC-UV and LC-MS," PLANTA MEDICA, vol. 78, no. 8, pp. 800-806, 2012.

[28] W. Wang, Q. Pan, X. Han et al., "Simultaneous determination of arctiin and its metabolites in rat urine and feces by HPLC," Fitoterapia, vol. 86, pp. 6-12, 2013.

[29] W. Xu, Y. Zhang, Y. Yu et al., "Dose-dependent target diversion of Danhong injection on the Glu-GLT-1/Gly-GlyR $\alpha$ dynamic balance module of cerebral ischemia," Pharmacological Research, vol. 135, pp. 80-88, 2018.

[30] H. Koskinen, H. Nordman, and B. Fröseth, “Serum lysozyme concentration in silicosis patients and workers exposed to silica dust," European Journal of Respiratory Diseases, vol. 65, no. 7, pp. 481-485, 1984.

[31] S. Beshir, H. Aziz, W. Shaheen, and E. Eltahlawy, "Serum levels of copper, ceruloplasmin and angiotensin converting enzyme among silicotic and non-silicotic workers," Open Access Macedonian Journal of Medical Sciences, vol. 3, no. 3, pp. 467-473, 2015.

[32] J. X. Shi and F. Pan, "Effect of pulmonary macrophage-derived lysozyme on the fibroblast growth," Journal of the Central University for Nationalities, vol. 92, no. 4, pp. 70-72, 2004.

[33] T. Wocka-Marek, M. Zajac-Nedza, U. Zygan, J. NiedzielaMarx, and A. Lukas, "Value of biochemical and immunologi- cal studies in the diagnosis of silicosis," Medycyna Pracy, vol. 42, no. 4, pp. 233-239, 1991.

[34] H. K. Gustavson, "The function of hydroxyproline in collagens," Nature, vol. 175, no. 4445, pp. 70-74, 1955.

[35] N. Y. Ignat'eva, N. A. Danilov, S. V. Averkiev, M. V. Obrezkova, V. V. Lunin, and E. N. Sobol', "Determination of hydroxyproline in tissues and the evaluation of the collagen content of the tissues," Journal of Analytical Chemistry, vol. 62, no. 1, pp. 51-57, 2007.

[36] X. X. Li, D. Y. Jiang, X. X. Huang, S. L. Guo, W. Yuan, and H. P. Dai, "Toll-like receptor 4 promotes fibrosis in bleomycin-induced lung injury in mice," Genetics and Molecular Research, vol. 14, no. 4, pp. 17391-17398, 2015.

[37] Z. He, Y. Gao, Y. Deng et al., "Lipopolysaccharide induces lung fibroblast proliferation through Toll-like receptor 4 signaling and the phosphoinositide3-kinase-Akt pathway," PLoS One, vol. 7, no. 4, p. e35926, 2012.

[38] H. Shen, N. Wu, Y. Wang et al., "MyD88 gene knockout attenuates paraquat-induced acute lung injury," Toxicology Letters, vol. 269, pp. 41-46, 2017.

[39] G. M. Barton and R. Medzhitov, "Toll-like receptor signaling pathways," Science, vol. 300, no. 5, pp. 1-8, 2014.

[40] J. Kuang, M. Xie, and X. Wei, "The NALP3 inflammasome is required for collagen synthesis via the NF- $\kappa \mathrm{B}$ pathway," International Journal of Molecular Medicine, vol. 4, no. 41, pp. 2279-2287, 2018.

[41] S. L. Cassel, S. C. Eisenbarth, S. S. Iyer et al., "The Nalp3 inflammasome is essential for the development of silicosis," Proceedings of the National Academy of Sciences of the United States of America, vol. 105, no. 26, pp. 9035-9040, 2008.

[42] B. T. Mossman, "Introduction to serial reviews on the role of reactive oxygen and nitrogen species (ROS/RNS) in lung injury and diseases," Free Radical Biology \& Medicine, vol. 34, no. 9, pp. 1115-1116, 2003.

[43] G. Bauernfeind Franz, G. Horvath, A. Stutz et al., "Cutting edge: NF- $\kappa \mathrm{B}$ activating pattern recognition and cytokine receptors license NLRP3 inflammasome activation by regulating NLRP3 expression," The Journal of Immunology, vol. 2, no. 183, pp. 787-791, 2009.

[44] C. Dostert, V. Petrilli, R. Van Bruggen, C. Steele, B. T. Mossman, and J. Tschopp, "Innate immune activation through Nalp3 inflammasome sensing of asbestos and silica," Science, vol. 320, no. 5876, pp. 674-677, 2008.

[45] D. Hanahan and R. A. Weinberg, "Hallmarks of cancer: the next generation," CELL, vol. 5, no. 144, pp. 646-674, 2011.

[46] J. Rastrick and M. Birrell, "The role of the inflammasome in fibrotic respiratory diseases," MINERVA MEDICA, vol. 105, no. 1, pp. 9-23, 2014.

[47] M. Sayan and B. T. Mossman, "The NLRP3 inflammasome in pathogenic particle and fibre-associated lung inflammation and diseases," Particle \& Fibre Toxicology, vol. 13, no. 1, p. $51,2015$.

[48] S. Ueha, F. H. W. Shand, and K. Matsushima, "Cellular and molecular mechanisms of chronic inflammation-associated organ fibrosis," Frontiers in Immunology, vol. 3, 2012.

[49] S. Liu, C. Hao, L. Bao, D. Zhao, and Y. Wu, "Silica particles mediate phenotypic and functional alteration of dendritic cells and induce Th2 cell polarization," Frontiers in Immunology, vol. 10, p. 787, 2019. 
[50] B. S. Franklin, M. S. Mangan, and E. Latz, "Crystal formation in inflammation," Annual Review of Immunology, vol. 34, no. 1, pp. 41015-55539, 2016.

[51] A. Desmouliere, "Transforming growth factor-beta 1 induces alpha-smooth muscle actin expression in granulation tissue myofibroblasts and in quiescent and growing cultured fibroblasts," Journal of cell biology, vol. 1, no. 122, pp. 103-111, 1993.

[52] B. S. Bagalad, K. P. M. Kumar, and H. K. Puneeth, "Myofibroblasts: master of disguise," Journal of Oral and Maxillofacial Pathology, vol. 21, no. 3, p. 462, 2017.

[53] A. L. Tatler and G. Jenkins, "TGF- $\beta$ activation and lung fibrosis," Proceedings of the American Thoracic Society, vol. 3, no. 9, pp. 130-136, 2012.

[54] I. A. Darby, N. Zakuan, F. Billet, and A. Desmoulière, "The myofibroblast, a key cell in normal and pathological tissue repair," Cellular and Molecular Life Sciences, vol. 73, no. 6, pp. 1145-1157, 2015.

[55] N. M. Green and D. A. Lowther, "Formation of collagen hydroxyproline in vitro," Biochemical Journal, vol. 71, no. 1, pp. 55-66, 1959.

[56] D. M. Brass, J. C. Spencer, L. Zhuowei et al., "Retraction: Innate immune activation by inhaled lipopolysaccharide, independent of oxidative stress, exacerbates silica-induced pulmonary fibrosis in mice," PLoS One, vol. 11, no. 5, p. e155388, 2016.

[57] L. G. Babushkina, B. A. Katsnel'Son, and N. S. Kisklitsina, "Effect of antioxidants on lipid metabolism and fibrogenesis disorders in lung tissue in experimental silicosis," Voprosy Meditsinskoi Khimii, vol. 27, no. 4, pp. 466-469, 1981.

[58] C. S. Jung, J.-S. Moon, K. Nikahira et al., “GLUT1-dependent glycolysis regulates exacerbation of fibrosis via AIM2 inflammasome activation,” Thorax, vol. 75, no. 3, p. 227, 2019.

[59] Q. Y. Xin, X. Y. Wan, and B. Y. Chen, "Effects of insulin-like growth factor-1 and insulin on modulating glucose metabolism and functions of lung fibroblasts," Chinese journal of tuberculosis and respiratory diseases, vol. 30, no. 8, pp. 605609, 2007.

[60] B. Zhang, Z. L. Chun, W. Xu, C. H. Xiang, and Y. F. Ma, "Luteolin alleviates NLRP3 inflammasome activation and directs macrophage polarization in lipopolysaccharidestimulated RAW264.7 cells," American Journal of Translational Research, vol. 10, no. 1, pp. 265-273, 2018.

[61] Z. Jie, X. Liu, C. Wan et al., "NLRP3 inflammasome mediates M1 macrophage polarization and IL- $1 \beta$ production in inflammatory root resorption," Journal of Clinical Periodontology, vol. 47, no. 4, pp. 451-460, 2020.

[62] L. Ying, X. Gao, Y. Miao et al., "NLRP3 regulates macrophage M2 polarization through up-regulation of IL-4 in asthma," Biochemical Journal, vol. 475, no. 12, pp. 1995-2008, 2018.

[63] J. Chen, H. Hou, H. Chen et al., "Urinary metabolomics for discovering metabolic biomarkers of laryngeal cancer using UPLC-QTOF/MS," Journal of Pharmaceutical \& Biomedical Analysis, vol. 167, pp. 83-89, 2019.

[64] E. Beauchamp, V. Rioux, and P. Legrand, "New regulatory and signal functions for myristic acid," médecine/sciences, vol. 25, no. 1, pp. 57-63, 2009.

[65] A. K. Srivastava, P. Khare, H. K. Nagar, N. Raghuwanshi, and R. Srivastava, "Hydroxyproline: a potential biochemical marker and its role in the pathogenesis of different diseases,"
Current Protein \& Peptide Science, vol. 17, no. 6, pp. 596602, 2016.

[66] Y.-j. Tan, Y.-s. Ren, L. Gao et al., "28-day oral chronic toxicity study of arctigenin in rats," Frontiers in Pharmacology, vol. 9, p. 1077, 2018.

[67] H. Bin, R. Zhi-ping, Z. Qi et al., "Study on acute toxicity of arctiin and burdock seed extract," Chinese Journal of Veterinary Drug, vol. 53, no. 7, pp. 44-48, 2019.

[68] L. Franchi, T. Eigenbrod, and G. Nunez, "Cutting edge: TNF- $\alpha$ mediates sensitization to ATP and silica via the NLRP3 inflammasome in the absence of microbial stimulation," Journal of Immunology, vol. 183, no. 2, pp. 792-796, 2009.

[69] T. Ozawa, "Studies on the component of the seed of Arctium lappa L. VI. The configuration of two asymmetric carbons of arctigenin and isoarctigenin," Yakugaku Zasshi Journal of the Pharmaceutical Society of Japan, vol. 3, no. 72, pp. 288-291, 1952.

[70] M. Jing-Yu, W. Zhi-Chao, and S. Chun-Qing, "Quantitative analysis of arctiin and arctigenin of Arctium Lappa L. from different areas by HPLC," Lishizhen Medicine and Materia Medica Research, vol. 15, no. 11, pp. 737-739, 2004.

[71] J. I. A. N. G. Hongshuai and D. O. U. Deqiang, "The biotransformation of arctiin by human intestinal bacteria and enzymes," Modern Chinese Medicine, vol. 16, no. 1, pp. 9-11, 2014.

[72] G. A. O. Qiong, Y. A. N. G. Mengbi, and Z. U. O. Zhong, "Overview of the anti-inflammatory effects pharmacokinetic properties and clinical efficacies of arctigenin and arctiin from Arctium Lappa L.," Acta Pharmacologica Sinica, vol. 39, no. 5, pp. 787-801, 2018. 